\title{
From complex questionnaire and interviewing data to intelligent Bayesian Network models for medical decision support
}

Anthony Costa Constantinou ${ }^{\mathrm{a}, \mathrm{c}}$, Norman Fenton`, William Marsh ${ }^{\mathrm{a}}$, and Lukasz Radlinski ${ }^{\mathrm{b}}$

a) Risk and Information Management Research Group, School of Electronic Engineering and Computer Science, Queen Mary University of London, Mile End Road, Mile End Campus, Computer Science Building, E1 4NS, London, UK.

b) Department of Software Engineering, West Pomeranian University of Technology, Szczecin ul. Żołnierska 52, 71-210 Szczecin, Poland

c) Corresponding author. E-mail address: anthony@constantinou.info

\section{THIS IS A PRE-PUBLICATION DRAFT OF THE FOLLOWING CITATION:}

Constantinou, A. C., Fenton, N., Marsh, W., \& Radlinski, L. (2016). From complex questionnaire and interviewing data to intelligent Bayesian Network models for medical decision support. To appear in Artificial Intelligence in Medicine, 2016.

DOI: $10.1016 /$ j.artmed.2016.01.002

Corresponding author: Dr. Anthony Constantinou, E-mail: anthony@constantinou.info

(C) 2016. This manuscript version is made available under the CC-BY-NC-ND 4.0 license: http://creativecommons.org/licenses/by-nc-nd/4.0/

\section{(cc) BY-NC-ND}




\begin{abstract}
Objectives: 1) To develop a rigorous and repeatable method for building effective Bayesian network (BN) models for medical decision support from complex, unstructured and incomplete patient questionnaires and interviews that inevitably contain examples of repetitive, redundant and contradictory responses; 2 ) To exploit expert knowledge in the BN development since further data acquisition is usually not possible; 3) To ensure the BN model can be used for interventional analysis; 4) To demonstrate why using data alone to learn the model structure and parameters is often unsatisfactory even when extensive data is available.
\end{abstract}

Method: The method is based on applying a range of recent BN developments targeted at helping experts build BNs given limited data. While most of the components of the method are based on established work, its novelty is that it provides a rigorous consolidated and generalised framework that addresses the whole life-cycle of BN model development. The method is based on two original and recent validated BN models in forensic psychiatry, known as DSVM-MSS and DSVM-P.

Results: When employed with the same datasets, the DSVM-MSS demonstrated competitive to superior predictive performance (AUC scores 0.708 and 0.797) against the state-of-the-art (AUC scores ranging from 0.527 to 0.705 ), and the DSVM-P demonstrated superior predictive performance (crossvalidated AUC score of 0.78 ) against the state-of-the-art (AUC scores ranging from 0.665 to 0.717 ). More importantly, the resulting models go beyond improving predictive accuracy and into usefulness for risk management purposes through intervention, and enhanced decision support in terms of answering complex clinical questions that are based on unobserved evidence.

Conclusions: This development process is applicable to any application domain which involves largescale decision analysis based on such complex information, rather than based on data with hard facts, and in conjunction with the incorporation of expert knowledge for decision support via intervention. The novelty extends to challenging the decision scientists to reason about building models based on what information is really required for inference, rather than based on what data is available and hence, forces decision scientists to use available data in a much smarter way.

Keywords: decision support, expert knowledge, Bayesian networks, belief networks, causal intervention, questionnaire data, survey data, mental health, criminology, forensic psychiatry. 


\section{Introduction}

Bayesian networks (BNs) are a well-established graphical formalism for encoding the conditional probabilistic relationships among uncertain variables of interest. The nodes of a BN represent variables and the arcs between variables represent causal, influential, or correlated relationships. The structure and the relationships in BNs can rely on both expert knowledge and relevant statistical data, meaning that they are well suited for enhanced decision making.

Underpinning $\mathrm{BNs}$ is Bayesian probability inference that provides a way for rational real-world reasoning. Any belief about uncertainty of some event $A$ is assumed to be provisional upon experience or data gained to date. This is what we call the prior probability, written $P(A)$. This prior probability is then updated by new experience or data $B$ to provide a revised belief about the uncertainty of $A$ that we call the posterior probability, written $P(A \mid B)$. The term Bayesian comes from Bayes' theorem which is a formula to determine $P(A \mid B)$ :

$$
P(A \mid B)=\frac{P(B \mid A) \times P(A)}{P(A)}
$$

Most real-world problems, including typically, medical risk assessment problems, involve multiple related uncertain variables and data, which are ideally represented as BNs. Early attempts to use Bayesian analysis in Artificial Intelligence applications to medical problems were unsuccessful due to the necessary Bayesian inference being, in general, computationally intractable [1]. However, the development of efficient BN inference propagation algorithms that work for large classes of practical BNs [2-4], along with advances in computational power over the last couple of decades, has caused a renewed interest in Bayesian probability for decision support. This has led to an enormous number of BN applications in a wide range of real-world problems [5] including, of course, medicine [6-9]. BNs are now being recognised as a powerful tool for risk analysis and decision support in real-world problems.

However, despite their demonstrable benefits, BNs still remain under-exploited, partly because there are no proven repeatable methods for their development when the development process requires the incorporation of expert knowledge due to limited or inappropriate data for inference. The problem is especially challenging when the only data available comes from poorly structured questionnaires and interviews involving answers to hundreds of relevant questions, but including inevitably examples of repetitive, redundant and contradictory responses. This is what we define as 'complex' data.

The objective of this paper is to propose a generic, repeatable, method for developing $\mathrm{BNs}$ by exploiting expert judgment and typically complex data that is common in medical problems. The method is specifically targeted to deal with the extremely common scenario, whereby the existing data cannot be extended except for the incorporation of expert knowledge. So there is no possibility of requesting data for either additional samples or additional variables. Essentially, we have to make the most of what we are given.

The method is derived from two case studies from the domain of forensic psychiatry. Specifically:

1. DSVM-P ("Decision Support Violence Management Prisoners"): a BN model for risk assessment and risk management of violent reoffending in released prisoners, many of whom suffer from mental health problems with serious background of violence [10];

2. DSVM-MSS ("Decision Support Violence Management Medium Security Services"): a BN model for violence risk analysis in patients discharged from medium security services [11].

Previously established predictive models in this area of research are either regression based or rule-based predictors, but their performance is poor and more importantly, they are incapable of simulating complex medical reasoning under uncertainty [10]. Hence, it was felt that BN models could improve on the state-of-the-art.

The two BN models were developed in collaboration with domain experts and the designers of the questionnaires. Both models demonstrated improved forecasting capability and enhanced usefulness for decision support (as we demonstrate in Section 9 and discuss in Sections 10 and 11) relative to the previous state-of-the-art models in this area of research.

However, in both cases we had to overcome the challenge of relying on patient data that had been collected before the use of BNs had been considered. As is typical with medical domain data much of it was 'complex', in the sense described above, coming from questionnaires and interviews with patients. The method described in this paper for developing BN models based on such existing complex data is an attempt to generalise what we did and learned in these forensic psychiatry applications.

While most of the components of the method are based on established work, its novelty is as follows: 
1. Provides a rigorous consolidated and generalised framework that addresses the whole life-cycle of BN model development for any application domain where there is constrained and complex data. Specifically where the problem involves decision analysis based on complex information retrieved from questionnaire and interview data, rather than based on data with hard facts, and in conjunction with the incorporation of expert knowledge.

2. Its starting point is an approach to problem framing that challenges decision scientists to reason about building models based on what information is really required for inference, rather than based on what data is available - even while it is assumed no new data can be provided. In other words, it forces decision scientists to use available data in a much smarter way.

The questionnaire and interviewing data, and the problems with learning from them, are discussed in Section 2 along with relevant literature review and a brief overview of the proposed method. Sections 3 to 8 describe the following respective steps of the method: Determine model objectives, Bayesian Network structure, Data Management, Parameter Learning, Interventional Modelling, and Structural Validation. Drawing on the case study results demonstrated in Section 9, we discuss the benefits and limitations of the method in Section 10, we provide a general discussion about the method and future research in Section 11, and we provide our concluding remarks in Section 12.

\section{The data and its problems}

As is typical for most medical BN building projects, in the forensic psychiatry studies we were presented with a set of unstructured patient data from questionnaires and interviews that had been collected independently of the requirements of a $\mathrm{BN}$ model. The questionnaires were large and complex and the data extracted from them was combined with other relevant patient data, such as criminal records, retrieved by the Police National Computer.

The questionnaire data includes patient responses to questions over the course of an interview with a specialist. They also include assessment data based on certain check-lists formulated by specialists, and which are taken into consideration for evaluating certain psychological and psychiatric aspects of the individual under assessment. The responses can take any form, from binary scale (such as Yes/No) and ordinal scale (such as Very low to Very high), to highly complicated multiple choice answers (with one or more possible selections), numerical answers (e.g. salary, number of friends), as well as free-from answers.

For example, in the DSVM-P study individuals were asked to complete up to 939 questions. All of the responses are coded in a database, and each response is represented by a variable. Since many of those questions were based on multiple choice answers (with up to approximately 20 choices), and with more than one answer being selected in most of the cases, the resulting database included a number of responses that was a multiple of the number of questions. As a result, there were thousands of variables in the relevant databases, excluding the data from criminal records retrieved by the Police National Computer. In the DSVM-MSS study, which was based on less extensive questionnaires, the total number of data variables was still well over 1,000.

Yet, despite the large number of variables, the databases in both studies had relatively small sample sizes (953 and 386 samples respectively for DSVM-P and DSVM-MSS) - again something that is very typical of many such studies. This makes them a poor starting point for developing effective BNs for decision-support and risk assessment, which normally require a very high ratio of samples to variables and/or substantial expert knowledge. This point is increasingly widely understood; we do not restrict the complexity of the model simply because we have limited or poor quality data $[12,13]$. BN applications which incorporate expert knowledge along with relevant statistical data have demonstrated significant improvements over models that rely only on what data is available; specifically in realworld applications requiring decision support [5, 14-18].

There have been limited previous attempts to develop BN models from questionnaire, interviewing or survey data:

1. Blodgett and Anderson [19] developed a BN model to analyse consumer complaints and concluded that the Bayesian framework offered rich and descriptive overview of the broader complaining behaviour process by providing insights into the determinants and subsequent behavioural outcomes, such as negative and positive word-of-mouth behaviour.

2. Sebastiani and Ramoni [20] developed a BN to analyse a dataset extracted from the British general household survey. The authors commented on the limitation of having to discretise all the data since continuous distributions were not supported by BN software at that time. 
3. Ronald et al. [21] found the following advantages of BNs (compared to more traditional statistical techniques) in analysing key linkages of the serviceprofit chain within the context of transportation service satisfaction: a) can provide causal explanation using observable variables within a single multivariate model, b) analyse nonlinear relationships contained in ordinal measurements, c) accommodate branching patterns that occur in data collection, and d) provide the ability to conduct probabilistic inference for prediction and diagnostics with an output metric that can be understood by managers and academics.

4. Salini and Kenett [22] examined BNs in analysing customer satisfaction from survey data with the intention of demonstrating the advantages of BNs in dealing with this type of data on the basis that "BNs have been rarely used so far in analyzing customer satisfaction data" [22].

5. Ishino [23] described a method of extracting knowledge from questionnaires for marketing purposes by performing BN modelling. This method was said to be a) capable of treating multiple objective variables in one model, b) handling nonlinear covariation between variables, and c) solving feature selection problems using Cramer's coefficient of association as an indicator [23]; though the benefits of (1) and (2) come as a result of using the $\mathrm{BN}$ framework.

With the exception of Ishino [23] the main focus of these previous studies were on the results and benefits of the developed BNs, rather than on the method of development. Moreover, all applications involved data from surveys and questionnaires for marketing and customer satisfaction purposes - generally a less complex application domain than medical. While Ishino [23], did focus on a method, it involved minimal expert input. Our focus is on a method for moving from the poorly structured, complex, but limited, data to an effective expert constructed BN model. Hence, we believe this is the first attempt to provide a whole-life cycle process for developing and validating $\mathrm{BN}$ models based on complex data and expert judgment.

The method is divided into six key component steps, as demonstrated by the iterative development process in Figure 1.

The following sections, from Section 3 to Section 8 , describe respectively the six steps. Throughout, we illustrate each step with examples from the two case studies and discuss the challenges for each development step in detail. 


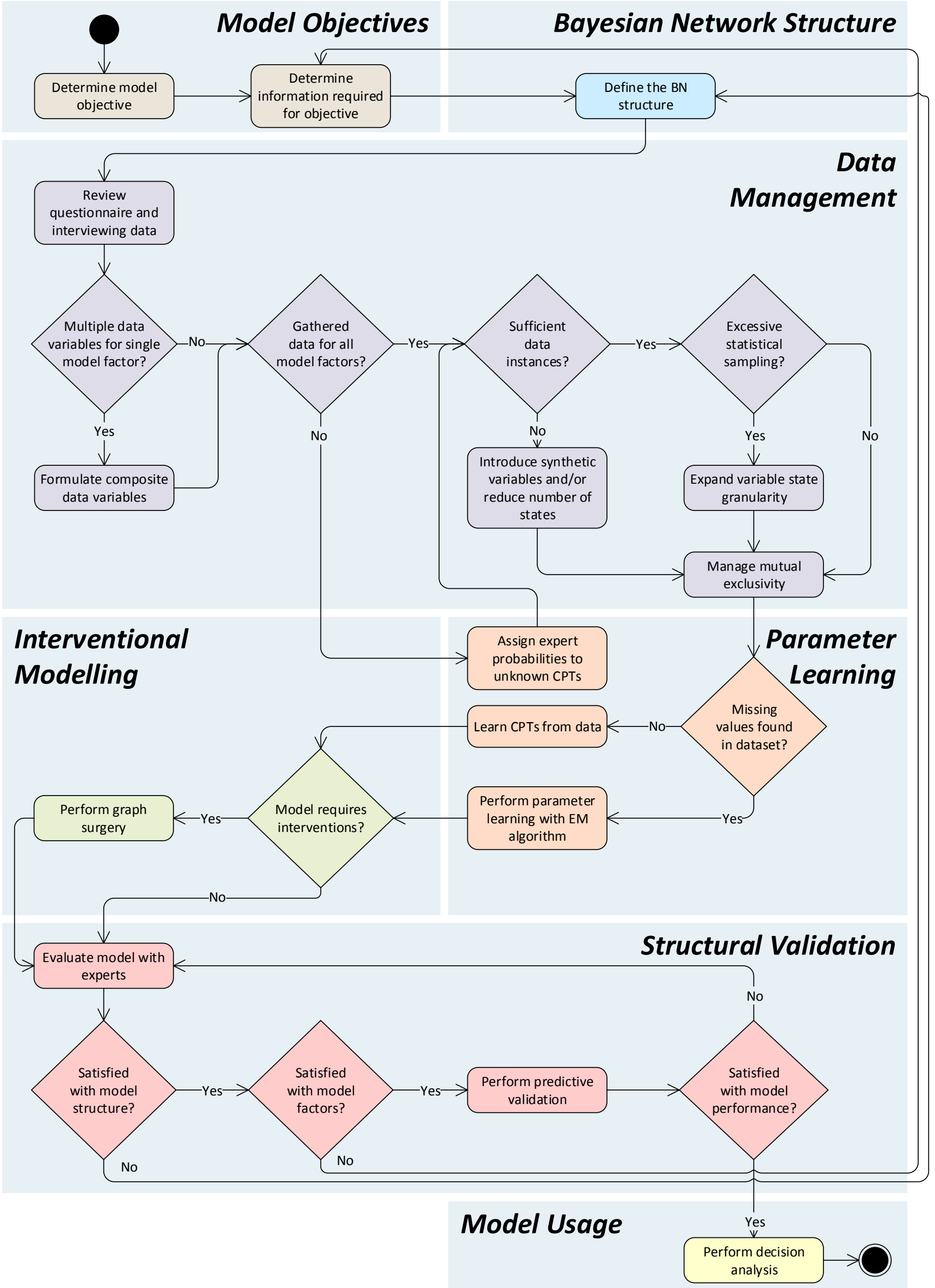

Figure 1. The proposed expert Bayesian network development process on the basis of learning from questionnaire interviewing data. 


\section{$3 \quad$ Model objectives}

The starting point of the method is the Model Objectives component. Although the availability of some existing patient/medical database is often the motivation to develop a BN ("we have this really great/important data we think you should be able to use it to build a BN model to support decision making for problem X...") it should never be the real starting point. This is true even in the scenario (which is the one we are assuming) whereby the available data cannot be extended except by expert knowledge. Instead, irrespective of whatever existing data is available (and certainly before even considering doing any kind of statistical analysis) the first step involves determining what the actual objective of the model is. For example, the following are very different classes of objectives for BN models:

1. Risk assessment: Determine the most likely current state of a variable (that is typically not directly observable). For example, "to determine from all the available information, the probability that a given person has disease $X$ " or "to determine if new drug $D$ is safe to use".

2. Risk Management: Determine the most likely outcome of some core variable for a given intervention action. For example, "What is the probability a patient's condition with respect to disease $X$ will improve if given treatment $T^{\prime \prime}$.

In the DSVM-P study the initial core objective was to determine if it is safe to release a given prisoner by assessing the prisoner's risk of violent reoffending in the case of release. Similarly, the core objective for the DSVM-MSS study was to determine if it is safe to discharge a given mentally ill patient by assessing the patient's risk of violence in the case of discharge. Both of these objectives represent a risk assessment process. But in both case studies, the objectives are expanded to risk management in the sense that the risk of violence for a given individual can be managed to acceptable levels after release/discharge by considering a number of relevant interventions (see Section 7).

Only when the objective is determined, can we specify what information we ideally require for carrying it out. Interviews with one or more domain experts are typically required in order to identify all of the important variables required to meet the core objective for the $\mathrm{BN}$ model. For our two BN applications, the domain experts were two clinical active experts in forensic psychiatry (Prof. Jeremy Coid) and forensic psychology (Dr. Mark Freestone) [10, 11]. In each case approximately five to seven meetings lasting between 1-2 hours with the domain experts were required at this stage in order to identify the important model factors (this really depends on domain complexity). In both studies, at least $75 \%$ of the model factors were identified at this initial stage.

The subsequent component of our proposed method is concerned with constructing a Bayesian network structure, in collaboration with domain experts, by considering the information that we really need to model.

\section{Bayesian network structure}

Assuming we have specified the 'ideal' required variables from the model objectives step, we can proceed into the most time consuming step of the process: constructing the structure of the BN model with expert knowledge. While BNs are often used to represent causal relationships between variables of interest, an arc from variable $A$ to variable $B$ does not necessarily require that $B$ is causally dependent on $A$ [13]. The 'ideal' variables constitute the initial set of nodes of the BN. Many BNs developed for medical real-world applications have been constructed by expert elicitation [6, 9, 24-28].

We do recognise that expert elicitation requires major interdisciplinary collaboration which can be complex and time consuming. In the DSVM-P study $75 \%$ of the model factors had been identified as a result of investigating what information we really require to meet the model objectives. It is only when the experts are involved in the design of the BN structure, and therefore start thinking in terms of dependency and/or cause and effect between factors, that they are able to identify the residual factors that were missed in the previous step.

Unlike the previous step, however, in the BN structure step the meetings were numerous and long. In DSVM-P there were around 20 meetings whose average time was approximately three hours. However, since we collaborated with the same experts for both case studies, the development of the BN structure for the second case study DSVM-MSS was approximately three to four times shorter than that of DSVM-P. We believe there were two reasons for this: 1) the experts had already 'learned' about both the process and BN models; and 2) there were generic similarities between the second and first study.

As noted in Figure 1, the BN structure we initially construct is likely to be quite different from the final version (as a result of subsequent iterations to model synthetic and mutually exclusive variables and also interventions). However, the conceptual flow of the network is likely to remain unchanged. Figures 2, 5 and 8 from respective Sections 5.2, 5.4 and 7 demonstrate how 
fragments of the $\mathrm{BN}$ model have been altered over the process as a result of introducing synthetic, mutual exclusive and interventional nodes in the BNs. The final versions of the two BNs are provided in Appendix A; Figures A1 and A2. The next component of our proposed method is concerned with mapping the data we actually have into the closest possible match to what we ideally need.

\section{Data management}

The primary objective of the data management task is to link actual data variables to model nodes. Because of the complexity of the data from questionnaires and interviews as described in Section 2, this is extremely challenging. Generally, there is no single data variable corresponding to an ideal' model variable. Typically there are multiple related data variables provided for similar questions. The challenge here mainly involves combining all these similar responses (which in some cases can also be inconsistent) into a single piece of information in an attempt to inform the relevant model node. These challenges are discussed in the following sub-subsections.

\subsection{Composite data variables}

The most common problem involves the need for a single variable which, although not in the data, has multiple associated variables. For example, in the DSVM-P study we had the following model nodes:

1. Financial difficulties: While there was no such variable in the available questionnaire data there was sufficient information to learn an approximate surrogate variable. Specifically, the sources of such relevant information are answers provided to questions such as "Are you behind paying bills?", "Have you recently had any services cut off?", and "What is your average weekly income".

2. Problematic life events: This was assessed on the basis of responses to questions such as "Separation due to marital difficulties, divorce or break down of steady relationship", "Serious problem with a close friend, neighbour or relative", and "Being made redundant or sacked from your job".

For both of these examples there were several more relevant sources of information that could have been considered to learn the specific model variables, and this was the case with many other model factors. As a result, problems arise in determining which data variable to choose for the particular node. Focusing on just one data variable is not expected to be the best approach since, in doing so, other relevant and important information will most likely be ignored.

A solution under these expert-driven circumstances is to formulate some combinational rule, or a set of combinational rules, for all the important data variables. We have worked with clinicians (psychiatrists and clinical psychologists) as well as the designers of the questionnaires themselves to retrieve the inferences we were interested in [10,11]. Examples of combinational rules between the different sources of similar information are:

1. an $O R$ relationship - i.e. Financial difficulties $=" Y e s "$ if at least one data variable satisfies this statement,

2. an AND relationship - i.e. Financial difficulties="Yes" if all the relevant data variables satisfy this statement,

3. a relative counter - i.e. Financial difficulties="Yes" if at least $X$ out of $Y$ data variables satisfy this statement,

4. a ranked average - i.e. Financial difficulties="Very high" if the majority of the data variables indicate severe financial difficulties,

5. a weighted ranked average - i.e. Financial difficulties="Very high" if the key data variables indicate severe financial difficulties.

Although many other combinational rules are possible, the five above should be enough to deal with the vast majority of these scenarios.

One class of cases, however, is especially problematic, namely where the data actually comprises records of expert knowledge. For instance, some records may reflect the clinician's assessment as to whether the individual suffers from a particular type of mental illness, or in identifying a certain type of behavioural attitude by interviewing the individual. In such situations we found it impractical to derive a clear-cut method of determining which combinational rules to use and when because the questionnaire and interviewing data was far too complex and uncertain. As a result, in these situations we required expert judgements to determine the necessary data sources and combinational rules. 


\subsection{Synthetic $B N$ nodes}

Although many relations in a $\mathrm{BN}$ can be causal, one of the most commonly occurring class of $\mathrm{BN}$ fragments is not causal at all. The definitional/synthesis idiom models this class of BN fragments.

A synthetic node is one which is simply defined by the values of its parent nodes, as demonstrated in Figure 2 (from the DSVM-MSS model), using some expert-driven combinational rule. Synthetic nodes can be extremely useful for:

1. reducing model dimensionality and the effects of combinatorial explosion, and

2. improving the overall $\mathrm{BN}$ structure of the model in terms of dependent/influential relationships.

In the case of (1), this vastly increases computational speed of the model and, crucially, reduces the size of the CPTs whose entries (i.e. parameters) have to be learned from data or elicited from experts (see Section 6). In the case of (2), while the synthetic nodes are not causally related to their parents (e.g. uncontrolled aggression summarises Aggression and Self-control), the network fragments themselves can help in constructing a BN model with more natural and dependency/influential relationships at the conceptual level (see Figure 2).

In the DSVM-MSS study the experts had initially suggested the eight specified variables with direct links to violence (Figure 2; top network) and this generated 1152 state combinations for the CPT of node Violence. This is clearly a problematic scenario, given that the sample size was just 386. Reconstructing this part of the network with the expertly defined synthetic nodes (Figure 2; bottom network) not only reduced the number of state permutations for the CPT of node Violence by $\sim 97 \%$ (i.e. down to 24 ), but also improved the conditional relationship between factors for violence risk analysis. Further, Table 1 presents, as an example, the CPT for the expertly defined synthetic node 'Uncontrolled aggression' introduced in the DSVM-MSS study. The expert reasoning is that if an individual does not have selfcontrol then we cannot expect the individual to be able to control his or her aggression, in the case of Aggression $=$ Yes, and vice versa.

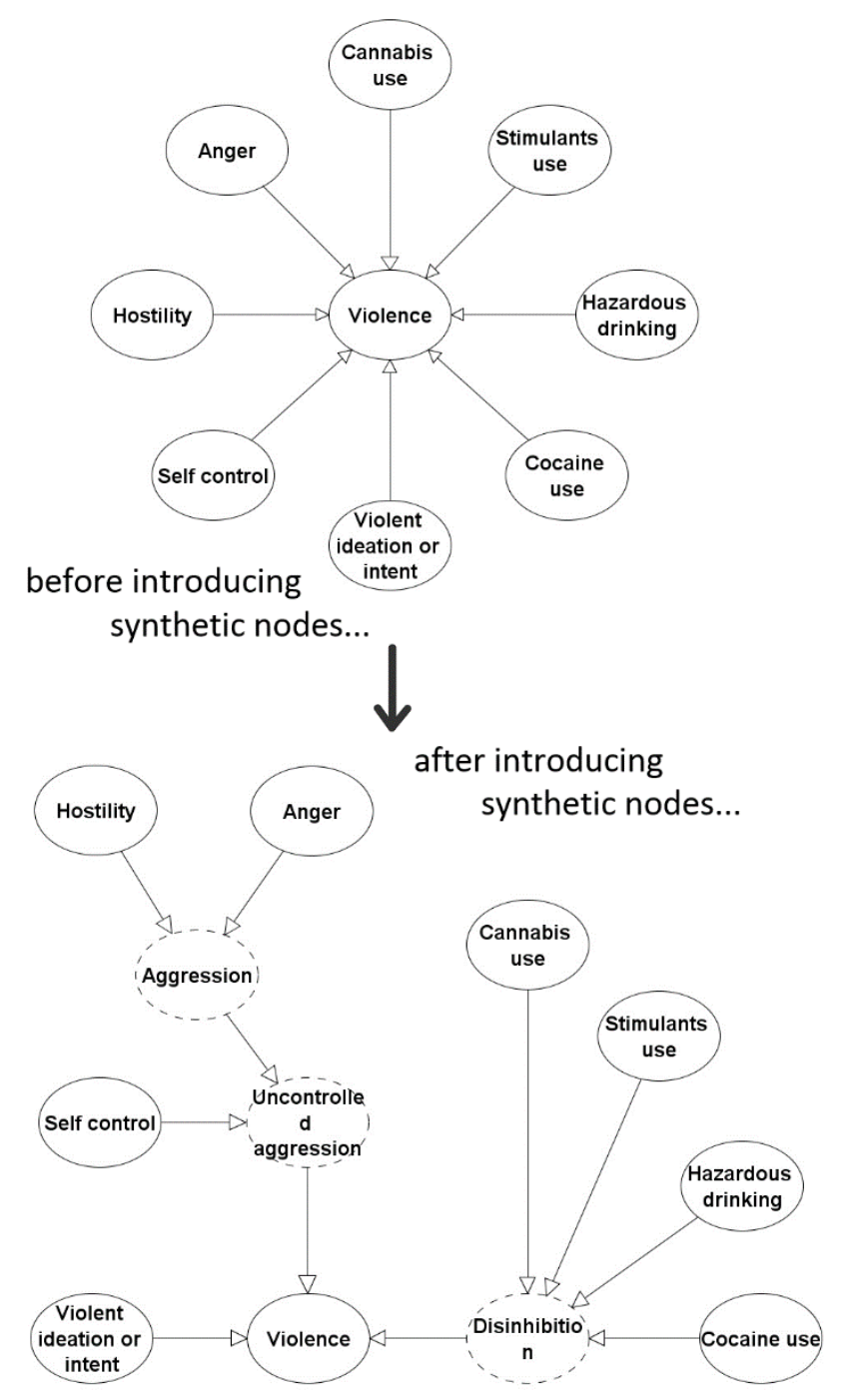

Figure 2. How the BN is revised after introducing synthetic nodes [11].

Table 1. Expertly defined CPT for synthetic node Uncontrolled Aggression.

\begin{tabular}{c|c|c|c|c|c|c}
\hline Self-control & \multicolumn{3}{|c|}{ No } & \multicolumn{3}{c}{ Yes } \\
\hline Aggression & No & Partly & Yes & No & Partly & Yes \\
\hline Low & 1 & 0 & 0 & 1 & 0 & 0 \\
High controlled & 0 & 0 & 0 & 0 & 1 & 1 \\
High uncontrolled & 0 & 1 & 1 & 0 & 0 & 0 \\
\hline
\end{tabular}

\subsection{Managing the number of states of the BN nodes}

Although many variables in the dataset are typically binary (i.e. Yes/No, True/False, High/Low), some have multiple states and some are continuous. For example, in the DSVM-P study we have 79 categorical nodes with the number of states ranging from two to nine, and nine continuous distribution nodes (four different types); whereas in the DSVM-MSS study we have 80 categorical nodes with the number of states ranging from two to eight. 
If we are learning the prior probabilities of the states from the data alone we need to ensure there not too many states relative to the sample size. If there are the learned probabilities will suffer from high variability, which typically results in model overfitting; i.e. leading to a model that performs well on the training data but poorly on unseen data. This happens when the model has not learned to generalise from trend. Depending on the parent nodes, sometimes even three states will be too many, while some variables may have up to $10+$ states. Under such circumstances, some sensible recategorisation of states must be performed in order to reduce the number of states for such variables.

Figure 3 illustrates a case from the DSVM-P study whereby we had to convert a Gaussian distribution of IQ scores into a categorical distribution consisting of six ordinal states. A quick look at the prior marginal probabilities of the categorical distribution, which appear to be normally distributed over the six states, as captured from data, provides us with confidence the size of data was sufficient for a reasonably well informed prior. Conversely, for the DSVM-MSS study the limited data restricted the number of states of the IQ node to just three. Appendix B, Table B.1 provides all the variables, from both models that have been downgraded in terms complexity in order to reduce the risk of model overfitting as a result of limited data.
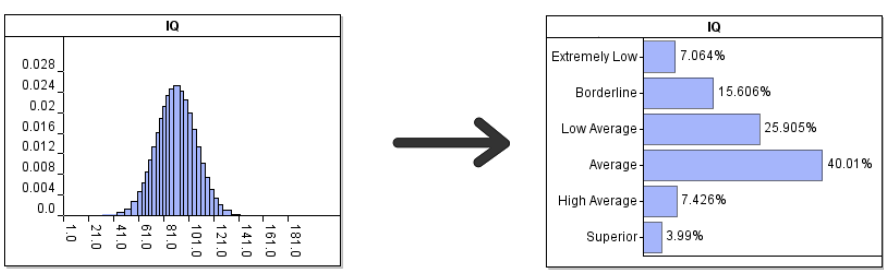

Figure 3. Converting a Gaussian distribution into a categorical distribution, as captured from data, with ordinal states. Note that the average IQ of the individuals in the study was below average.

When the states of the variable are known to follow an ordinal scale distribution, but the dataset is not sufficiently large to capture the normality as accurate as that of Figure 3, other approaches can be considered such as Ranked nodes in BNs which are ordinal categorical distributions generated on the basis of Truncated Gaussian distributions [29]. Figure 4 demonstrates how the same Gaussian distribution from Figure 3 can be converted into a Ranked distribution of the same six states by normalising the mean and variance into a truncated version with lower and upper boundaries set to 0 and 1 respectively; effectively a TruncatedGaussian [0,1] distribution as proposed by [29].

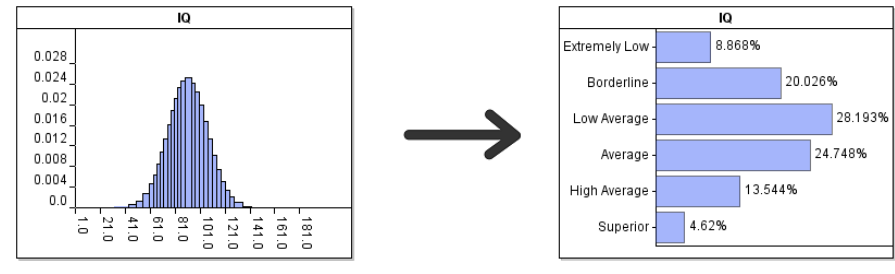

Figure 4. Converting a Gaussian distribution into a Ranked distribution based on the mean and variance of the Gaussian distribution, as proposed by Fenton et al. [29].

Properly managing the type of nodes (i.e. categorical/continuous), the number of node states, and the type of states (i.e. nominal/ordinal), can dramatically help in increasing computational speed while concurrently improving the model's predictive accuracy.

\subsection{Mutual exclusivity}

Datasets resulting from questionnaires and interviews will likely incorporate multiple variables that are mutually exclusive. Such variables can usually be more simply modelled in a BN as the set of states of another single generalised variable (by definition such states are mutually exclusive).

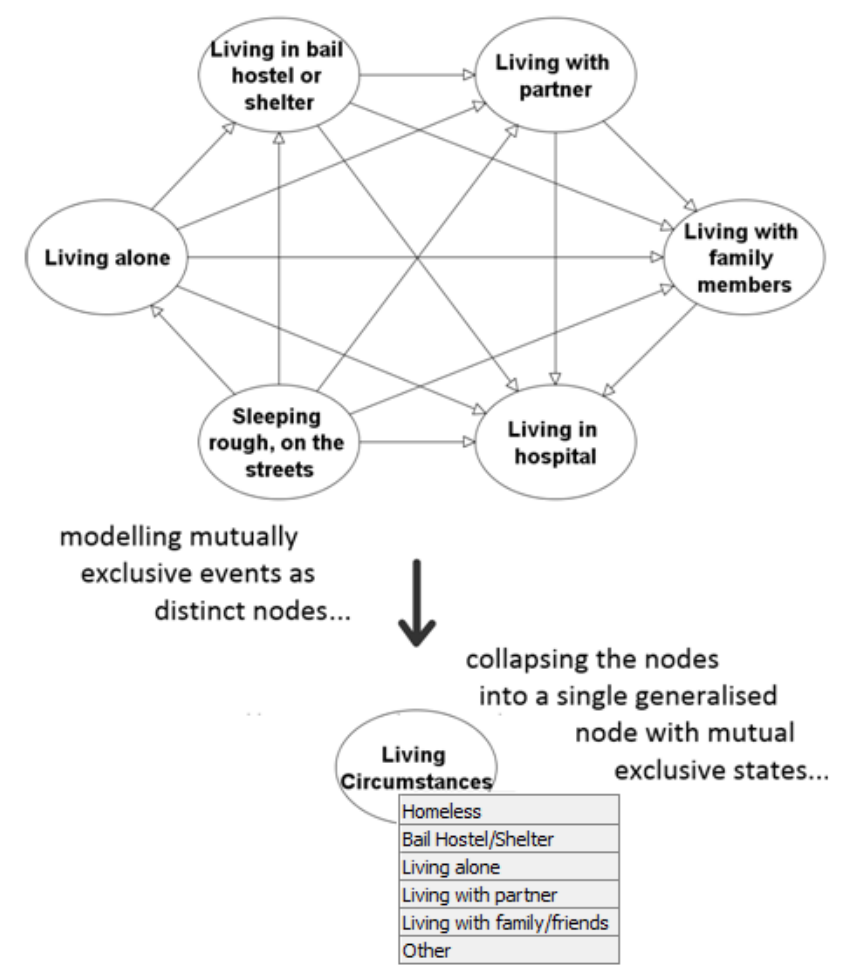

Figure 5. Collapsing mutual exclusive data variables into a single generalised node with mutual exclusive states.

An example of this common phenomena arising in the DSVM-P study is shown in Figure 5. Here there is a set of seven mutually exclusive Boolean variables in the 
dataset (there were many more but the experts identified these seven to be sufficient and the most important); they can be collapsed into a single generalised categorical node. This assumes that all of the mutual exclusive variables share identical parent and child nodes, and are therefore not required to be modelled as distinct nodes [30]. Properly managing mutual exclusivity reduces model complexity, makes parameter learning and elicitation simpler, and increases computational speed.

\section{Parameter learning}

Parameter learning is the process of determining the CPT entries for each node of the agreed $\mathrm{BN}$ model. It is expected to be performed once the model structure is stable and all of the data management issues have been satisfactorily addressed

Because of the limitations of the real-world data, even allowing for the methods described in Section 5, there will generally be nodes or individual parameter values, for which no relevant data is available. For these cases, we chose to elicit the probability values from the domain experts (in the DSVM-P study four out of 89 of the nodes required expert elicitation, while in the DSVM-MSS study it was six out of 80 such nodes). We address this process in Section 6.1. Alternatively, there are data-driven techniques which could be considered for finding reasonable assignments to missing variables, and this is covered in Section 6.2 where we describe the method for learning the CPTs from data.

\subsection{Expert-driven learning}

Various expert-driven probability elicitation methods have been proposed. However, most of them are similar and rather simple as they tend to propose some sort of probability scale with verbal and/or numerical anchors, as well as focusing on speeding up the elicitation process as it can sometimes be a daunting task [27, 31-33].

The expert-driven probability elicitation process we considered for both case studies was similar to those referred above, using verbal representations for probability scale such as from Very low (i.e. 0 to 0.2 ) to Very high (i.e. 0.8 to 1). We also endeavoured to keep the questions put to experts as simple as possible; at no point were the expert asked to combine multiple pieces of uncertain information in their head in order to arrive at a conclusion.

We ensured that domain experts would only be required to answer straightforward questions such as: "How strong is the influence between $A$ and B?", or "how high is the risk of treatment $Y$ causing a side-effect?". We also found helpful the following studies [5, 34-37] which, in addition to providing further recommendations on eliciting expert probabilities, also provide guidelines on how to minimise bias during the elicitation process.

\subsection{Data-driven learning}

Unfortunately, in both our studies the vast majority of the data-driven variables had missing data. The only data-driven nodes with complete data were those based on criminal data provided by the Police National Computer (e.g. Age, Gender, Number of violent convictions). This is typical of real-world data in medical domains where it is generally accepted that patient data collected during the course of clinical care will inevitably suffer from missing data [38].

In dealing with datasets which include missing data, decision scientists typically have three options [39]:

1. Restrict parameter learning only to cases with complete data: For the reasons explained above this is not a viable option for typical medical studies.

2. Use imputation-based approaches: In these missing values are filled with the most probable value, based on the values of known cases, and then the CPTs are learned normally as if they were considering a full dataset. There are multiple imputation methods; for example, the imputed value can be chosen based on the mean predicted value when considering all of the other know values, or a subset of them, or even based on regression procedures [40].

3. Use likelihood-based approaches: In these the missing values are inferred from existing model and data (i.e. the model attempts to infer the likelihood of missing values that make the observed data most likely). The Expected Maximisation (EM) algorithm, which is an iterative method for approximating the values of missing data [41], is commonly used for this purpose, and is widely accepted as the standard approach for dealing with missing data in BNs.

In both studies we chose option (3) and the EM algorithm to learn the CPTs of variables which are based on data with missing values. Specifically, the EM algorithm is based on forming the conditional expectation of the log-likelihood function for completed data given the observed data as defined in [41]:

$$
Q\left(\theta^{\prime} \mid \theta\right)=E_{\theta}\left\{\log f\left(X \mid \theta^{\prime}\right) \mid y\right\}
$$


where $X$ is the random variable which corresponds to the complete data, which is unobserved, with density $f$, and $y=g(x)$ is the observed data. The log-likelihood function for the complete data is a linear function of the set of sufficient marginals:

$$
n\left(i_{a}\right), \quad a \in A, \quad i_{a} \in I_{a} .
$$

The EM algorithm searches for the Maximum Likelihood Estimate (MLE) of the marginal likelihood by iteratively applying the following two steps:

1. Expectation $(E)$ step. This calculates the expected value of the log-likelihood function and which is equivalent to calculating the expected marginal counts:

$$
n^{*}\left(i_{a}\right)=E_{p}\left\{N\left(i_{a}\right) \mid \text { observed data }\right\}
$$

2. Maximisation $(M)$ step. This solves:

$$
n\left(i_{a}\right)=n^{*}\left(i_{a}\right), \quad a \in A, \quad i_{a} \in I_{a},
$$

for $p$ which, assuming the expected counts were the true counts, maximises the likelihood function.

For this task, we have made use of the EM learning engine offered by the freely available GeNIe [42]. This is because GeNIe offers the two following important features during the learning process:

1. Fixed nodes: During the parameter learning process, expert-driven variables must be indicated as Fixed nodes in order to retain their prior probabilities as suggested by the experts; assuming that no data exist for these variables that will allow EM algorithm to generate meaningful inferences (see Figure 6 for an example);

2. Confidence: If the EM algorithm is used to revise a previously learned model with a new (additional) relevant dataset, then a level of confidence should be assigned to the prior probabilities of the input model. The confidence expresses the level of certainty in the parameters assigned to the local probability distributions in the input model. Specifically, it represents the number of cases/records the original parameters are based on.

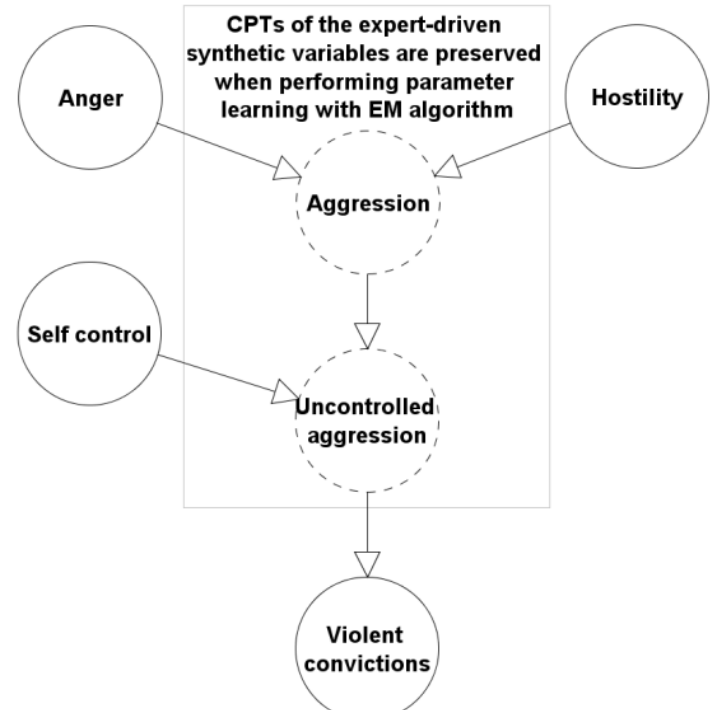

Figure 6. A small BN fragment from the DSVM-MSS indicating the CPTs of expert-driven synthetic variables are preserved after performing parameter learning with EM algorithm.

\section{Interventional modelling}

Just like other probabilistic models, a BN model can be used to inform how probabilities for uncertain events are expected to change (e.g. for the purposes of risk assessment) based on a number of other relevant events that are known within that model. The causal framework, which represents both BNs as well as Influence Diagrams (IDs), makes these models particularly suitable in informing how the probabilities would also change as a result of some intervention (i.e. for the purposes of risk management).

IDs have traditionally been the preferred type of probabilistic graphical model for decision support problems since, unlike $\mathrm{BNs}$, they allowed the user to incorporate decision as well as utility nodes, in additional to chance nodes, for solving decision problems. More specifically, while a chance node represents a random variable, a decision node represents the options that are available to the decision maker, whereas utility nodes represent the decision maker's preferences [43]. However, recent work on interventions (discussed below) allows the decision maker to model decisions or actions in the form of an intervention in $\mathrm{BNs}$, and determine their value based on their influence against some desired output variable, which can be anything from a Boolean variable to a continuous distribution. Previous work mainly focuses on perfect interventions; i.e. when the effect variable is set to a single state following the intervention [13, 44-46]. The process of intervening on an event that is rendered independent of all its causes is known as graph surgery [13]. 


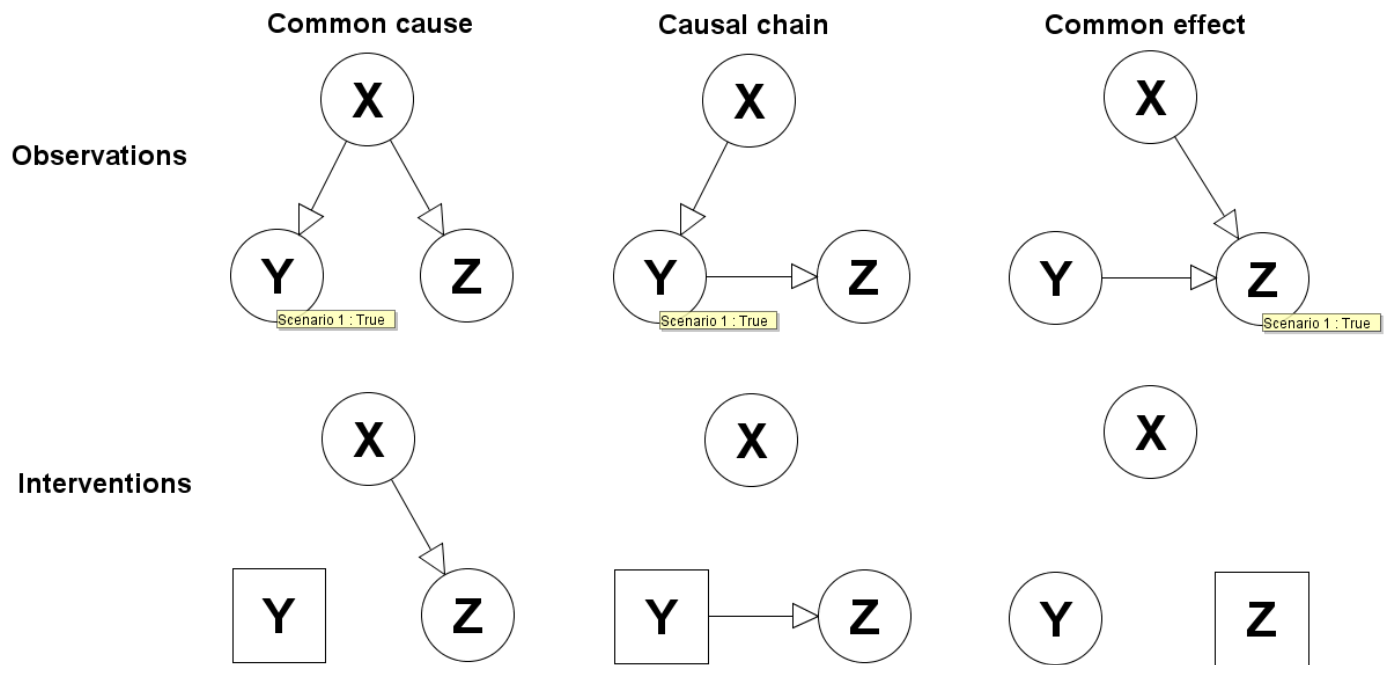

Figure 7. The three basic DAG models with examples of observations, which are represented by the nodes set to True, and interventions, which are represented by squared nodes [46].

Hagmayer et al [46] illustrate this concept on the three basic directed acyclic graph (DAG) models presented in Figure 7, where:

1. Common cause: $P(X, Y, Z)=P(Y \mid X) P(Z \mid X) P(X)$;

2. Causal chain: $P(X, Y, Z)=P(Z \mid Y) P(Y \mid X) P(X)$;

3. Common effect: $P(X, Y, Z)=P(Z \mid Y, X) P(Y) P(X)$;

Figure 7 illustrates the concept of graph surgery when it comes to perfect interventions by demonstrating how the links between variables are removed when modelling an intervention, represented by square nodes in bottom models, rather than an observation (i.e. top models). For a more detailed description see [13].

In medical informatics, however, an intervention is typically represented by some sort of treatment, therapy or medication, and is typically used to answer questions such as: "If a patient receives treatment $X$, what are the chances of him getting well?". This would represent an imperfect intervention; implying that the intervention induces a distribution over states rather than a specific state $[47,48]$. Our focus is on this more complex type of intervention.

By definition, the effectiveness of an imperfect intervention depends on some other factors. We identified two such factors in our case studies:

1. the individual's motivation for treatment, and

2. the individual's responsiveness to treatment.
The purpose of these two additional factors is to influence the effectiveness of any relevant imperfect intervention. These additional factors are described as switch nodes in [48].

Figure 8 presents an example, based on the DSVM-MSS study, of how an observational BN model is expected to transform into an interventional $\mathrm{BN}$ model in order to allow execution of imperfect interventions. We will discuss these two steps in turn:

1. Observational model: When learning the CPTs of the model parameters, no interventions are taken into consideration. At this phase, the 'Treatment for anger' is simply an observation; e.g. what is the probability that the doctor will propose treatment for anger, given anger.

2. Interventional model: When the model is used for intervention we alter the original model by removing any arcs entering the desired interventions (i.e. arcs with an $X$ in Figure 8). The dependency links are removed because in this scenario we do not want to explain the observation for treatment, but rather to estimate its impact as an intervention; hence, we must not reason backwards diagnostically.

This example also demonstrates that it is possible for the intervention to serve as the child node of the relevant symptom in observational models, but this link should be reversed (if not removed) in the interventional model. Specifically, while in the observational model we expected evidence of Anger to increase the chance for a doctor to propose Treatment for anger, in the interventional model we 
would expect Treatment for anger to reduce symptoms of Anger.

Further, the switch nodes in this example are Motivation to attend treatment and Responsiveness to treatment. As a result, both of these factors influence Anger post-treatment in the interventional phase since the effectiveness of Treatment for anger is dependent upon them.

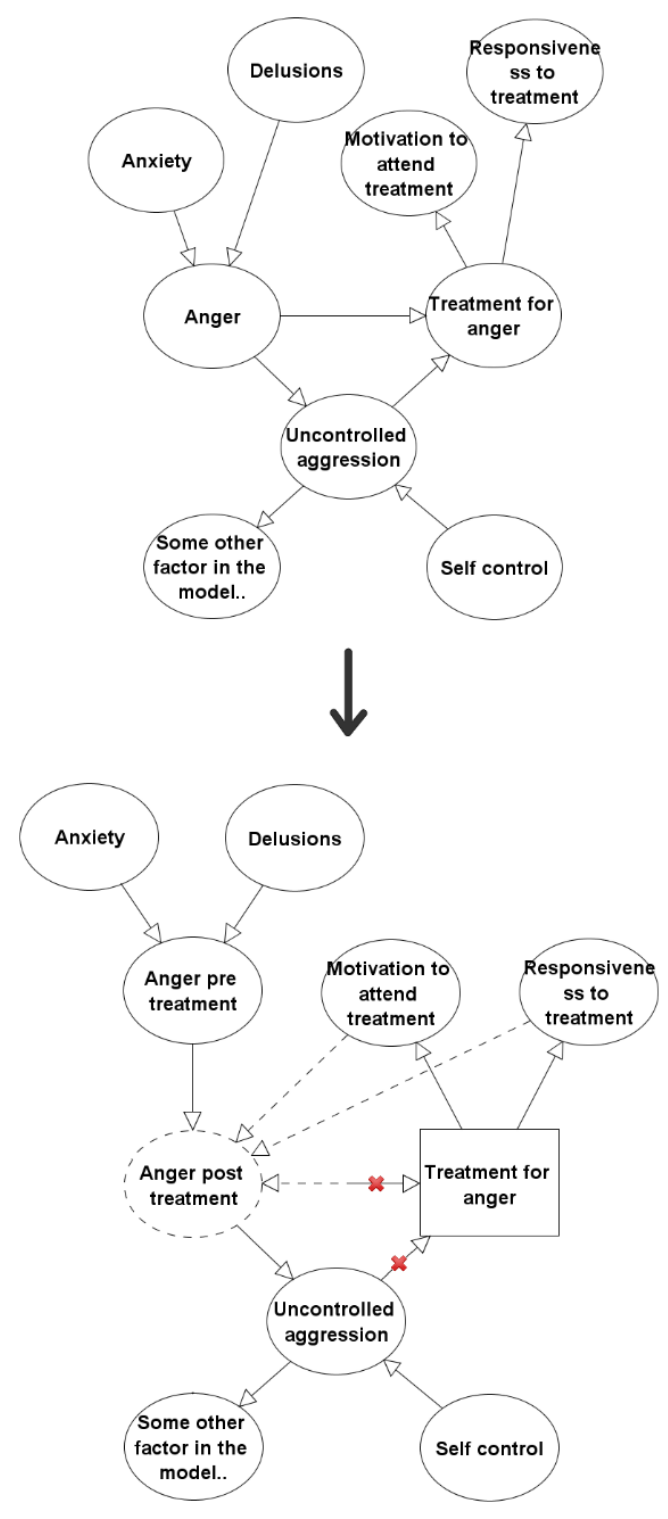

Figure 8. An example of how an observational BN model transforms into an interventional BN model, on the basis of imperfect interventions. Dashed nodes and dashed arcs are introduced in the interventional phase.

In both of our studies, there was data providing follow-up information about the patients/prisoners for up to five years post-discharge/release. This provided us with information about the effectiveness of the relevant treatments and therapies and allowed us to estimate the effect of interventional actions from data. Fortunately, even when no such data is available, BNs will allow us to formulate interventions and define their impact based on expert knowledge.

For a more detailed discussion, including some real-world experiments, on the distinction between observational and interventional probabilistic relations see [46]. Further, with imperfect information models it is possible to have more than one intervention manipulating the same symptom. The model presented in Figure 8 allows for such multiple interventions. This process has also been described as fat hand intervention model [48].

\section{Structural validation}

In terms of validating the structure of the $\mathrm{BN}$ model, apart from predictive accuracy, we found sensitivity analysis (SA) to be particularly useful. SA is a simple, yet powerful, technique for examining the impact of specified model factors against a desired targeted factor within the same model. This is achieved by recalculating the outcomes of the targeted factor under alternative assumptions. It is possible to see diagrammatically which nodes, and under what states, have the greatest impact on any selected target node as we subsequently show in Figure 9. We used the freely available AgenaRisk software for the sensitivity analysis since this process is fully automated in that package [49].

SA can serve as an extremely useful tool for rapidly evaluating:

1. The structure and the CPTs of the BN: The sensitivity results depend on the structure of the model and hence, the overall robustness of a BN model can be swiftly assessed with domain experts, in an attempt to identify possible irrationalities for both the BN structure and the underlying CPTs [50, 51];

2. Interventional structure and effectiveness: The sensitivity results also depend on which set of model factors are instantiated (different sets of node instantiations will normally lead to different sensitivity scores). Consequently, we can also use SA to validate interventional structure with domain experts and to assess the effectiveness of each intervention.

Furthermore, the validation that SA can provide extends to risk management assessment of individual scenarios. For instance, and based on our application domain, SA can be used with a set of observations that represent a patient's profile, in order to assess potential revisions with regards to the risk of violence and on the 
basis of some intervention. As an example, Figure 9 presents the tornado graph ${ }^{1}$ generated for risk management purposes, based on a prisoner's profile from our case studies. The effectiveness of the three specified interventions is assessed against the prisoner's profile, and which indicates that the individual:

1. suffers from mental illness,

2. is drug dependent,

3. is alcohol dependent,

4. is partly impulsive,

5. has no violent thoughts,

6. is motivated to attend treatments,

7. is responsive to treatments and therapies

For instance, the graph indicates that if we set Psychiatric treatment $(\mathrm{P})$ to "No", we get $p($ Violence $=Y e s)=0.661$, whereas if we enable this particular intervention the respective risk of violence drops down to $0.534^{2}$.

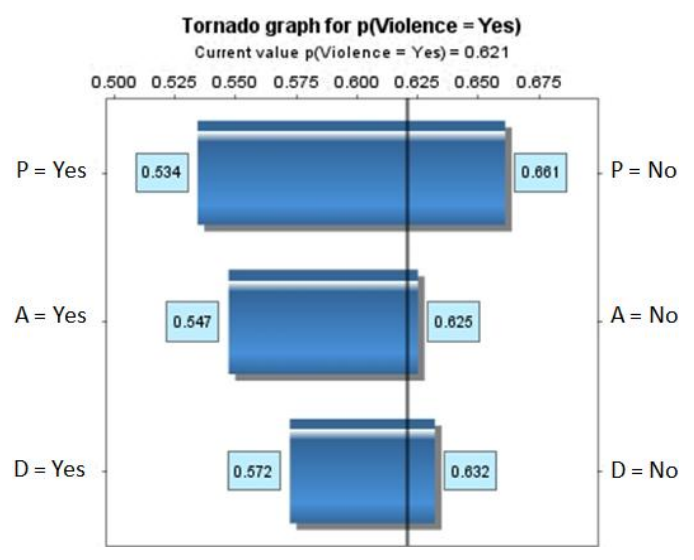

Figure 9. Sensitivity analysis for the three specified interventions, on the risk of observing violence over a specified time post-release, based on a made-up profile of an individual (discussed in text); where $P$ is Psychotic treatment, $A$ is Alcohol treatment, and $D$ is Drug treatment.

\section{Results from validation and predictive accuracy}

The accuracy of the two BN models was validated on the basis of cross-validation and with respect to whether a prisoner/patient is determined suitable for

\footnotetext{
1 The graph is generated using the AgenaRisk BN simulator [49].
}

2 SA assumes that residual interventions remain uncertain. It requires that the factors provided as an input for SA are uncertain. For a more accurate assessment for each individual intervention, SA should be performed only based on a single intervention (with the residual interventions disabled). However, SA is not capable of examining the effectiveness of interventions when they are combined (i.e. when more than one intervention is active). To achieve this, the decision maker must manually perform this observations in the network and record the alteration of probabilities on the target variable. In [62] we demonstrate how the underlying principle of Value of Information can enhance decision analysis in uncertain BNs with multiple interventions. release/discharge, using the area under the curve (AUC) of a receiver operating characteristic (ROC) [52]. The AUC of ROC was considered simply because, in these application domains, it represents the standard method for assessing binary predictive distributions. This allowed us to perform direct comparisons, in terms of AUC scores, against the current state-of-the-art models developed for violence risk assessment and prisoners' release decision making.

The well-established models and predictors for which we base our comparisons against are either regression-based models, or rule-based techniques with no statistical composition. Specifically:

1. HCR20v3 [53] and HCR-20v2 [54]: are Structured Professional Judgment (SPJ) assessment tools developed based on empirical literature review factors that relate to violence. They are used primarily by clinicians seeking to assess readiness for discharge amongst patients whose mental disorder is linked to their offending. A total of 20 Items are scored on a three-point scale by clinicians.

2. SAPROF [55]: is 17-item checklist-scale where items are scored on the same trichotomous scale as the HCR-20. The items are grouped into internal (e.g. mental), external (e.g. environmental) and motivational (e.g. incentives) factors.

3. PANSS [56]: is a 30-item evaluation scale that focuses on measuring the severity of symptoms of mental illness. The symptoms are groups into positive (i.e. outwardly displayed symptoms associated with psychosis), negative (i.e. relating to diminished volition and self-care), general (i.e. non-specific symptoms) and aggression.

4. VRAG [57]: is a regression-based model based on 12 variables linked to violence and which correlate best with reoffending.

5. PCL-R [58]: is a checklist of 20 variables which measure psychopathy, and which are strongly related with offending behaviour in prisoner populations.

The DSVM-MSS model was assessed against the 12 predictors shown in Figures 11 and 12, and in terms of both General violence (i.e. minor violent incidences) and Violent Convictions (i.e. major violent incidences). The DSVM-P model was assessed against three predictors shown in Figure 12, and in terms of Violent Convictions. Table 2 provides a summary of the results. 
Table 2. Predictive validation for DSVM-MSS and DSVM-P, based on the AUC of ROC, where ' $<$ ' represents the number of models for which the specified BN model performed significantly inferior, ' $=$ ' represents the number of models for which no significant differences have been observed in predictive accuracy, and ' $>$ ' represents the number of models for which the specified BN model performed significantly superior. Significant levels for DSVM-MSS were set to 0.05, whereas for DSVM-P were set to 0.001 .

\begin{tabular}{|c|c|c|c|c|c|c|}
\hline Model & Validated outcome & $\begin{array}{c}\text { Post-discharge } \\
\text { period }\end{array}$ & $\begin{array}{c}\text { Validated against } \\
X \text { models }\end{array}$ & $<$ & $=$ & $>$ \\
\hline \multirow[t]{2}{*}{ DSVM-MSS } & $\begin{array}{l}\text { General violence } \\
\quad(\mathrm{AUC}=0.708)\end{array}$ & 12 & $X=13$ & 0 & $\begin{array}{c}10 \\
\text { (AUCs between } \\
0.626 \text { and } 0.705 \text { ) }\end{array}$ & $\begin{array}{c}3 \\
\text { (AUCs between } \\
0.549 \text { and } 0.622 \text { ) }\end{array}$ \\
\hline & $\begin{array}{l}\text { Violent convictions } \\
\quad(\mathrm{AUC}=0.797)\end{array}$ & months & $X=13$ & 0 & $\begin{array}{c}9 \\
\text { (AUCs between } \\
0.622 \text { and } 0.685 \text { ) }\end{array}$ & $\begin{array}{c}4 \\
\text { (AUCs between } \\
0.527 \text { and } 0.614 \text { ) }\end{array}$ \\
\hline DSVM-P & $\begin{array}{l}\text { Violent convictions } \\
\quad(\mathrm{AUC}=0.78)\end{array}$ & $\begin{array}{l}1816 \\
\text { days }\end{array}$ & $X=3$ & 0 & 0 & $\begin{array}{c}3 \\
\text { (AUCs between } \\
0.665 \text { and } 0.717 \text { ) }\end{array}$ \\
\hline
\end{tabular}

Overall, the DSVM-MSS model demonstrated competitive predictive capability, whereas the DSVM-P model demonstrated superior predictive capability, when compared against the current state-of-the-art predictors that are employed with the same dataset. More specifically:

1. DSVM-MSS and General Violence: Figure 10 illustrates that the AUC score of the DSVM-MSS model matches the best scores generated by the thirteen predictors specified and, as shown in Table 2, significantly outperforms three out of the thirteen predictors. Overall, the DSVM-MSS model in this case demonstrated competitive performance against the current state-of-the-art.

2. DSVM-MSS and Violent Convictions: Figure 11 illustrates that the AUC score of the DSVM-MSS model outperforms all the other scores generated by the thirteen predictors specified and, as shown in Table 2, significantly outperforms four out of the thirteen predictors. Overall, the DSVM-MSS model in this case demonstrated competitive to superior performance against the current state-of-the-art.

3. DSVM-P and Violent Convictions: Figure 12 illustrates that the AUC score of the DSVM-P model outperforms all the other scores generated by the three predictors specified and, as shown in Table 2, significantly outperforms all of the predictors. Overall, the DSVM-P model demonstrated superior performance against the current state-of-the-art.

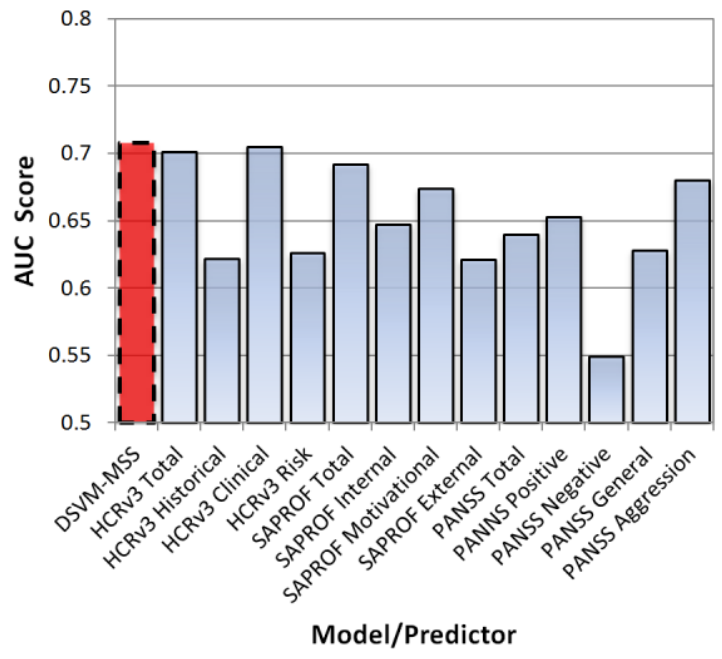

Figure 10. AUC score comparison, in terms of predicting incidences of General Violence, between DSVM-MSS and the specified 13 well known predictors, when employed with the same dataset.

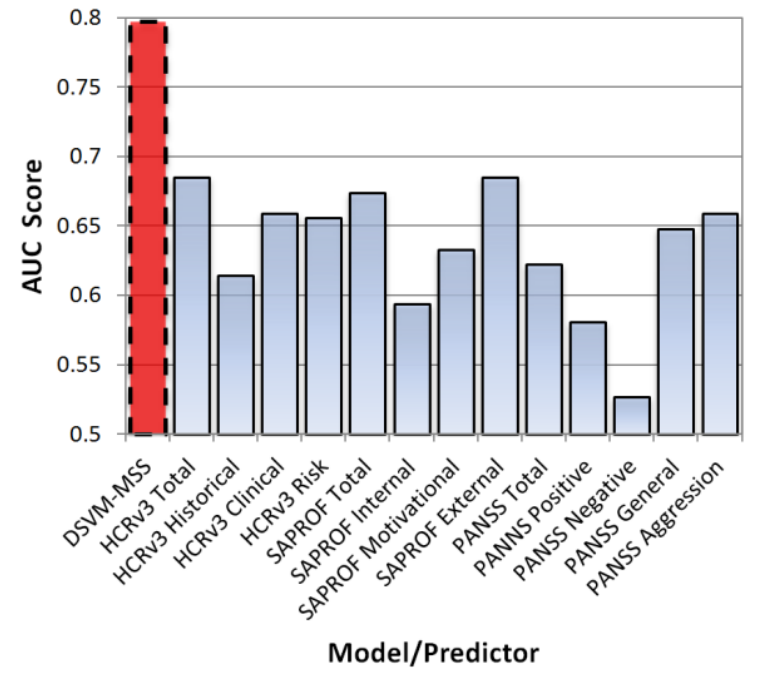

Figure 11. AUC score comparison, in terms of predicting incidences of Violent Convictions, between DSVM-MSS and the specified 13 well known predictors, when employed with the same dataset. 


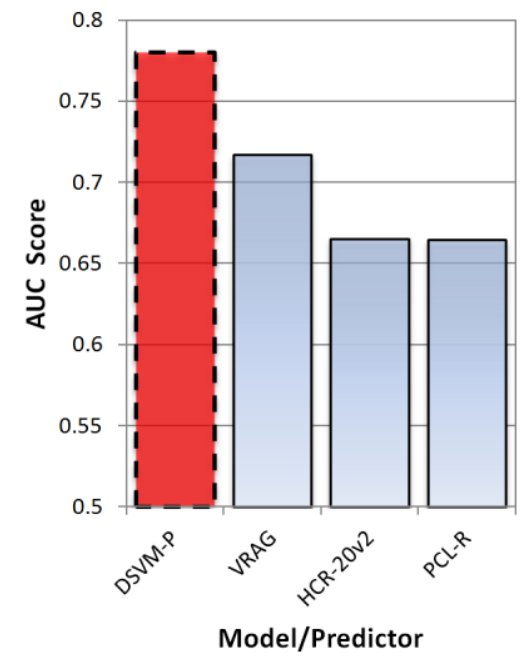

Figure 12. AUC score comparison, in terms of predicting incidences of Violent Convictions, between DSVM-P and the specified three well known predictors, when employed with the same dataset.

\section{Discussion}

We first summarise the benefits and limitations of the method presented in the paper as informed from the two case study models. Some of the benefits and limitations are clearly quite general in the sense that they apply to a wide range of other medical decision support problems.

The two case study BN models compare favourably against the well-established predictors in this area of research, which are based on either regression models or even some rule-based methods with no statistical composition, and which represent the current state-ofthe-art. Specifically, the BN models enhance decision support as follows:

1. Improved accuracy: The DSVM-MSS demonstrated competitive predictive capability whereas DSVM-P demonstrated superior predictive capability, when compared to the current state-of-the-art predictors that are employed with the same dataset.

2. Interventional analysis: The $\mathrm{BN}$ approach allows for specific factors to be targeted for intervention for risk management purposes. In the case of the two case studies, this is done by examining whether the risk of future re-offending or violent behaviour can be managed to acceptable levels as a result of some intervention (e.g. treatment/therapy), and this makes the model useful in terms of answering complex clinical questions that are based on unobserved evidence.

3. Inverse inference: Contrary to the current state-ofthe-art predictors, the $\mathrm{BN}$ framework allows for inference to be performed from both cause to effect and vice versa. This unique capability, also known as explaining away, can be used in the case study models by professionals to examine the reasons as to why a particular individual reoffended when the model may have been suggesting otherwise.

4. Handles missing evidence: Consider a prisoner and/or a mental health patient who does not respond to all of the questionnaire/interviewing questions. While current predictors only consider what information is available for prediction, the $\mathrm{BN}$ models allow flexibility with model inputs due to the BN framework; implying that missing inputs are not ignored simply because relevant evidence are not available, but rather inferred from other relevant evidence within the model.

5. Structural integrity based on expert knowledge: The BNs represent the most widely accepted technique for incorporating expert knowledge along with relevant historical data. Since expert knowledge can be easily incorporated into these kind of models, future relevant studies may choose to retain the proposed structure of these models (even at the conceptual level) regardless of how limited the dataset might be in terms of the number of variables.

On the other hand, the limitations are:

1. Extensive effort required for development: Developing expert-driven BN models not only requires collaboration with domain experts but also an extensive iterative development process. In case study DSVM-P the overall effort spanned just over a year and in the DSVM-MSS study, which followed the DSVM-P study, just below six months. Even though the method presents and covers a range of techniques for reducing the burden of expert elicited models, this up-front development effort remains the primary barrier to more widespread adoption of BNs.

2. Necessary use of subjectivity: The method and the resulting BNs models heavily rely on expert knowledge. Notwithstanding the various techniques inserted to avoid known biases for expert knowledge, it is impossible to avoid them completely, which inevitably assume subjectivity as well as possible bias. Involving multiple experts over the development process minimises the risk of bias, but further increases the effort required for development. 
3. Complexity: The proposed method should lead to a minimum number of variables and a conceptually well-structured and rational model. However, because the method encourages incorporation of expert-driven variables (which are additional to those in the dataset) there is a risk that experts will over-complicate the model, adding multiple layers of detailed variables.

Having identified the important limitations of depending on expert knowledge in almost all of the development stages, we need to justify its usefulness. It is important to note that, with the advent of 'big data' much of the current research on $\mathrm{BN}$ development assumes that sufficient data are available to learn the underlying BN structure, hence making the expert's input minimal or even redundant. For example, we could have made use of:

1. algorithms designed for parameter learning with insufficient and/or imbalanced data [59];

2. BN learning methods that are appropriate for use with small datasets but which include a large number of variables [60];

Making use of such algorithms eliminates, or minimises, the requirement for expert elicitation. This is very convenient in the sense that a $\mathrm{BN}$ model can be generated without much effort since we can skip the process of knowledge elicitation, which is extremely time consuming since it typically requires collaboration with multiple domain experts.

Conversely, the method illustrated in this paper involves extensive use of expertise, in almost all of the development stages, which greatly increases the effort required. This is because:

1. Modelling information that matters: We propose that the starting point of a decision support model is to determine what information we really require for inference, rather than generating a model based on what data is available. This is particularly important for the two case studies covered in this paper. This is because the available data is mostly represented by responses to questions rather than hard facts, and this causes numerous other decision support problems (see points 3 and 4 below).

2. Poor quality data means meaningless BN structure: As discussed in Section 2, our case studies were based on datasets consisting of thousands of variables, but the sample sizes of those variables was below 1,000. To address this problem, the experts identified (in each case study) less than 100 model factors as a requirement in order to construct a comprehensive BN.

On the other hand, if we were to make use of a structure learning algorithm we would have ended up with a extensively large network of associations between hundreds/thousands of responses as recorded from questionnaires and interviews. Even when these available responses represent information that associates with all of the ideal variables identified for inference, there is typically far too many variables and far too few samples in many medical applications to achieve any sensible structural learning with the state-of-the-art algorithms; especially in the case of complex and imbalanced data [61].

Using a structure learning algorithm in these scenarios results in models that may be superficially objective, but with a BN structure that is optimised for some features in the data. This is especially problematic when the structure is learned on biased datasets, which is a common challenge in healthcare settings with well-known inconsistencies in recording data.

3. Interventional modelling and risk management: A number of interventions are typically available to the clinicians and probation officers for managing relevant risks of interests. The resulting $\mathrm{BNs}$ from this study provide this capability to the decision makers based on the framework described in Section 7.

A BN model learned purely from data in these scenarios will fail to capture the necessary underlying dependency structure in situations where interventions and controls for risk management are not captured by historical data. However, even if the historical data captures factors that represent interventions, this process still requires careful elicitation of expertise. This is because we require the expert/s to indicate which of the variables represent actual interventions. Furthermore, interventions need to satisfy specific structural-rules (e.g. Graph surgery and uncertain interventions). On the basis of uncertain interventions, we also require expertise to identify the variables which are responsible for the uncertainty of an intervention (e.g. responsiveness to treatment and motivation for treatment). Furthermore, if we were to generate a BN model from data, we would have ended up simulating interventions on questionnaire and interviewing 
responses, rather than on more meaningful variables of interest.

4. Counterfactual modelling: Counterfactual analysis enables decision makers to compare the observed results in the real world to those of a hypothetical world. That is, what actually happened and what would have happened under some different scenario. While counterfactual analysis is out of the scope of this paper, it is worth mentioning that this type of analysis requires further use of expertise, for counterfactual modelling purposes, as demonstrated in [62], and on the basis of the application domains considered in this paper.

\section{Future work}

The method is expected to be applicable to any other application domain which involves making inferences from data records which represent responses from questionnaires, surveys and interviews. For example, marketing is an area where questionnaire and survey data, as well as free-form data from focus groups and individual interviews is extensive. Furthermore, just like the medical domains, marketing decision making also involves critical intervention actions as covered in the proposed method. The method presented in this paper will help in describing a more general method to systemise the development of effective BNs for decision analysis in all of those common situations where there is limited or complex data but access to expert knowledge.

However, in domains such as cancer and bioinformatics it can be much more complex to retrieve relevant information from an expert and hence, under such cases there is an increased risk of a weakly defined $\mathrm{BN}$ model. As a result, for future research we are also interested in investigating ways to minimise expert dependency. One possible direction is to enhance structure learning algorithms, which allow for constrains based on expert knowledge [16-18, 63], with systematic rules for interventional risk management and decision analysis.

Furthermore, our future research directions include describing a more formal approach to generic problem framing that seeks to minimise model redundancy in conjunction with efficient use of expert knowledge and data. A formalised tool will also be developed to support these enhancements. These generic problems are being addressed in the BAYESKNOWLEDGE project [64].

\section{Conclusions}

We have presented a generic, repeatable method for developing real-world $\mathrm{BN}$ models that combine both expert knowledge and data, when (part of) the data is based on complex questionnaires and interviews with patients that is available in medical problems.

The method is described in six primary steps: a) Model objectives, b) BN structure, c) Data management, d) Parameter learning, e) Interventional modelling, and f) Structural validation. We have demonstrated how the incorporation of expert knowledge, along with relevant historical data, becomes necessary in an effort to provide decision makers with a model that goes beyond the predictive accuracy and into usefulness for risk management through intervention and enhanced decision support.

While most of the components of the method are based on established work, the novelty of the method is that it provides a rigorous consolidated and generalised framework that addresses the whole life-cycle of $\mathrm{BN}$ model development. This development process is applicable to any application domain which involves decision analysis based on complex information, rather than based on data with hard facts, and in conjunction with the incorporation of expert knowledge for decision support via intervention. The novelty extends to challenging the decision scientists to reason about building models based on what information is really required for inference, rather than based on what data is available.

While the method requires an extensive iterative process between decision scientists and domain experts, BNs clearly offer potential for transformative improvements. The up-front development effort remains the primary barrier to more widespread adoption of BNs. The method presents and covers a range of techniques for reducing the burden of expert elicited models, and planned research directions will investigate ways to minimise expert dependency without damaging the decision support benefits illustrated in this paper.

Although the method is the primary contribution, it is important to note that the resulting $\mathrm{BNs}$ in the case studies are, to our knowledge, the first instances of $\mathrm{BN}$ models in forensic psychiatry for the purposes of violence prevention management in the decision making of released prisoners and mentally ill patients discharged from MSS.

In validating the method, we have shown that while both $\mathrm{BN}$ applications provide improvements in predictive accuracy against the current state-of-the-art, an equally important contribution is the usefulness the models provide in terms of decision support (an 
increasingly important criteria for models in medical informatics). Although the method was proposed and evaluated in a forensic medical setting, it is still expected to be applicable to any other real-world scenario, such as marketing, where BN models are required for decision support, where a) part of the data is based on complex questionnaire, survey, and interviewing data, and b) decision making involves the simulation of interventions on inferences as generated on the basis of such complex data, and in conjunction with expert knowledge.

\section{Acknowledgements}

We acknowledge the financial support by the European Research Council (ERC) for funding this research project, ERC-2013-AdG339182-BAYES_KNOWLEDGE, and Agena Ltd for software support. We also acknowledge the expert input provided by Dr Mark Freestone and Professor Jeremy Coid in developing the two Bayesian network applications, which are based on the method presented in this paper, but which are described in two other papers $[10,11]$.

\section{References}

[1] Cooper, G. F. (1990). The computational complexity of probabilistic inference using Bayesian Belief Networks. Artificial Intelligence, 42(2-3), 393-405.

[2] Pearl J. (1988). Probabilistic reasoning in intelligent systems: networks of plausible inference. San Mateo, CA: Morgan Kaufmann Publishers.

[3] Heckerman, D. E., Mamdani, A., \& Wellman, M. P. (1995). RealWorld Applications of Bayesian Networks - Introduction. Communications of the ACM, 38: 24-6.

[4] Jensen, F. V. (1996). An Introduction to Bayesian Networks. Springer, New York.

[5] Fenton, N.E., \& Neil, M. (2012). Risk Assessment and Decision Analysis with Bayesian Networks. Chapman and Hall, London.

[6] Heckerman, D. E., Horvitz, E. J., Nathwani, B. N. (1992). Towards normative expert systems. I. The Pathfinder project. Methods of Information in Medicine, 31: 90-105.

[7] Díez, F. J., Mira, J., Iturralde, E., \& Zubillaga, S. (1997). DIAVAL, a Bayesian expert system for echocardiography. Artificial Intelligence in Medicine, 10 (1): 59-73.

[8] Nikovski, D. (2000). Constructing Bayesian networks for medical diagnosis from incomplete and partially correct statistics. IEEE Transaction on Knowledge and Data Engineering, 12, 4: 509-516.

[9] Yet, B., Perkins, Z., Fenton, N., Tai, N. \& Marsh, W. (2013). Not just data: A method for improving prediction with knowledge. Journal of biomedical informatics, 48:28-37.
[10] Constantinou, A. C., Freestone, M., Marsh, W., Fenton, N. \& Coid, J. (2015). Risk assessment and risk management of violent reoffending among prisoners. Expert Systems with Applications, 42(21): 7511-7529.

[11] Constantinou, A. C., Freestone, M., Marsh, W., \& Coid, J. (2015). Causal inference for violence risk management in Forensic Psychiatry. Decision Support Systems, 80: 42-55.

[12] Spiegelhalter, D. J., Abrams, K. R., \& Myles, J. P. (2004). Bayesian Approaches to Clinical Trials and Health-care Evaluation. John Wiley and Sons, England.

[13] Pearl J. (2009). Causality: Models, Reasoning and Inference. 2nd edition, Cambridge University Press, UK.

[14] Constantinou, A. C., Fenton, N. E. \& Neil, M. (2012). pi-football: A Bayesian network model for forecasting Association Football match outcomes. Knowledge-Based Systems, 36: 322, 339.

[15] Constantinou, A. C., Fenton, Norman E. \& Neil, M. (2013). Profiting from an inefficient Association Football gambling market: Prediction, Risk and Uncertainty using Bayesian networks. Knowledge-Based Systems, 50: 60-86.

[16] Zhou, Y., Fenton, N., \& Neil, M. (2014). Bayesian network approach to multinomial parameter learning using data and expert judgments. International Journal of Approximate Reasoning, 55(5), 1252-1268

[17] Zhou, Y., Fenton, N. E., \& Neil, M. (2014). An Extended MPL-C Model for Bayesian Network Parameter Learning with Exterior Constraints. In L. van der Gaag \& A. J. Feelders (Eds.), Probabilistic Graphical Models: 7th European Workshop. PGM 2014, Utrecht. The Netherlands, September 17-19, 2014 (pp. 581-596). Springer Lecture Notes in AI 8754.

[18] Zhou, Y., \& Fenton, N. E. (2015). An Empirical Study of Bayesian Network Parameter Learning with Monotonic Causality Constraints. International Journal of Approximate Reasoning, In press 2015.

[19] Blodgett, J. G., \& Anderson, R. D. (2000). A Bayesian Network Model of the Consumer Complaint Process. Journal of Service Research, 2 (4): 321-338.

[20] Sebastiani, P., \& Ramoni, M. (2001). On the use of Bayesian networks to analyze survey data. Research in Official Statistics, 4 (1): 53-64.

[21] Ronald, A., Mackoy, R., Thompson, V. B., \& Harrell, G. (2004). A Bayesian Network Estimation of the Service-Profit Chain for Transport Service Satisfaction. Decision Sciences, 35 (4): 665-689.

[22] Salini, S., \& Kenett, R. S. (2009). Bayesian networks of customer survey satisfaction survey data. Journal of Applied Statistics, 36 (11): 1177-1189.

[23] Ishino, Y. (2014). Knowledge Extraction of Consumers' Attitude and Behavior: A Case Study of Private Medical Insurance Policy in Japan. The 8th International Conference on Knowledge Management in Organizations. Springer Proceedings in Complexity, 425-438.

[24] Heckerman, D. E., \& Nathwani, B. N. (1992). Towards normative expert systems. II. Probability-based representations for efficient 
knowledge acquisition and inference. Methods of Information in Medicine, 31: 106-16.

[25] Andreassen S., Riekehr C., Kristensen B., Schønheyder, H. C., Leibovici L. (1999). Using probabilistic and decision theoretic methods in treatment and prognosis modelling. Artificial Intelligence in Medicine, 15: 121-34.

[26] Lucas, P. J. F., De Bruijn, N. C., Schurink, K., \& Hoepelman, I. M. (2009). A probabilistic and decision-theoretic approach to the management of infectious disease at the ICU. Artificial Intelligence in Medicine, 19 (3): 251-79.

[27] van der Gaag, L. C., Renooij, S., Witteman, C. L., Aleman, B. M., Taal, B. G. (2002). Probabilities for a probabilistic network: a case study in oesophageal cancer. Artificial Intelligence in Medicine, 25: 123-148.

[28] Yet B., Bastani K., Raharjo H., Lifvergren S., Marsh W., \& Bergman B. (2013). Decision Support System for Warfarin Therapy Management using Bayesian Networks. Decision Support Systems, 55(2): 488-498.

[29] Fenton, N., Neil, M., \& Caballero, J. G. (2007). Using Ranked nodes to model qualitative judgments in Bayesian Networks. IEEE Transactions on Knowledge and Data Engineering, 19 (10): 1420-1432.

[30] Nielsen, T. D., \& Jensen, F. V. (2007). Bayesian networks and decision graphs. Springer-Verlag New York.

[31] Kuipers, B., Moskowitz, A. J., Kassirer, J. P. (1988). Critical decisions under uncertainty: Representation and structure. Cognitive Science, 12: 177-210.

[32] van der Gaag, L. C., Renooij, S., Witteman, C. L. M., Aleman, B., \& Taal, B. G. (1999). How to elicit many probabilities. In: Proceedings of the 15th International Conference on Uncertainty in Artificial Intelligence, San Francisco, CA: Morgan Kaufmann, 647-54.

[33] Renooij, S. (2001). Probability elicitation for belief networks: issues to consider. Knowledge Engineering Review, 16 (3): 255-69.

[34] Druzdzel, M. J., \& van der Gaag, L. C. (1995). Elicitation of Probabilities for Belief Networks: Combining Qualitative and Quantitative Information. Proc 11th Ann Conf on Uncertainty in Artificial Intelligence (UAI-05), 141-148, Montreal, Quebec, Canada, August.

[35] O'Hagan, A., Buck, C. E., Daneshkhah, A., Eiser, J. R., Garthwaite, P. H., Jenkinson, et al. (2006). Uncertain Judgments: Eliciting Experts' Probabilities. Wiley, Chichester.

[36] Johnson, S. R., Tomlinson, G. A., Hawker, G. A., Granton, J. T., Grosbein, H. A., \& Feldman, B. M. (2010). A valid and reliable belief elicitation method for Bayesian priors. Journal of Clinical Epidemiology, 63(4): 370-383.

[37] Hubbard, D., \& Drummond, D. (2014). How to Measure Anything: Finding the Value of "Intangibles" in Business. Wiley, New York.

[38] Lin, J., \& Haug, P. (2008). Exploiting missing clinical data in Bayesian network modelling for predicting medical problems. Journal of Biomedical Informatics, 41: 1-14.

[39] Little, R. J.A., \& Rubin, D. B. (2002). Statistical analysis with missing data. 2nd Edition. Wiley-Interscience, New Jersey.
[40] Enders, C. K. (2006). A primer on the use of modern missing-data methods in psychosomatic medicine research. Psychosomatic Medicine, 68:427-36.

[41] Lauritzen, S. L. (1995). The EM algorithm for graphical association models with missing data. Computational Statistics \& Data Analysis, 19: 191-201.

[42] GeNIe \& SMILE. (1998). Graphical Network Interface GeNIe \& SMILE. https://dslpitt.org/genie/index.php/about (Accessed: 3 November, 2015).

[43] Lacave, C., Luque, M., \& Diez, F. J. (2007). Explanation of Bayesian Networks and Influence Diagrams in Elvira. IEEE Transactions on Systems, Man., and Cybernetics - Part B: Cybernetics, 37 (4): 952-965.

[44] Spirtes, P., Glymour, C., \& Scheines, R. (1993). Causation, prediction, and search. Springer, New York.

[45] Woodward, J. (2003). Making things happen. A theory of causal explanation. Oxford University Press,.

[46] Hagmayer, Y., Sloman, S. A., Lagnado, D. A., \& Waldmann, M. R. (2007). Causal reasoning through intervention. In Causal learning: Psychology, philosophy and computation, ed. A. Gopnik \& L. Schulz. Oxford University Press, Oxford, England.

[47] Korb, K., Hope, L., Nicholson, A., \& Axnick, K. (2004). Varieties of causal intervention. In Pacific Rim Conference on AI, 2014.

[48] Eaton, D., \& Murphy, K. (2007). Exact Bayesian structure learning from uncertain interventions. In AI \& Statistics, 2007.

[49] Agena. (2015). Agena: Bayesian Network and Simulation Software for Risk Analysis and Decision Support. http://www.agenarisk.com (Accessed: 7 November, 2015).

[50] Coupe, V. M. H., \& van der Gaag, L. C. (2000). Sensitivity analysis: an aid for probability elicitation. Knowledge Engineering Review, 15: $215-32$.

[51] van der Gaag, L. C., \& Renooij, S. (2001). Analysing sensitivity data. In: Proceedings of the 17th International Conference on Uncertainty in Artificial Intelligence. San Francisco, CA: Morgan Kaufmann, 530-7.

[52] Hanley, J. A., \& McNeil, B. J. (1982). The meaning and use of the area under a receiver operating characteristic (ROC) curve. Radiology, 143: 29-36.

[53] Douglas, K. S., Hart, S. D., Webster, C. D., \& Belfrage, H. (2013). HCR-20V3: Assessing Risk of Violence - User Guide. Mental Health, Law, and Policy Institute, Simon Fraser University, Burnaby, Canada.

[54] Webster, C.D., Douglas, K.S., Eaves, D.E. \& Hart, S.D. (1997). HCR20: Assessing Risk for Violence, Version 2. Simon Fraser University, Vancouver, BC.

[55] de Vries Robbé, M., de Vogel, V., \& de Spa, E. (2011). Protective factors for violence risk in forensic psychiatric patients. A retrospective validation study of the SAPROF. International Journal of Forensic Mental Health, 10, 178-186.

[56] Kay, S.R., Fiszbein, A., \& Opler, L.A. (1987). The Positive and Negative Syndrome Scale (Panss) for Schizophrenia. Schizophrenia Bulletin, 13, 261-276. 
[57] Quinsey, V.L. (1998) Violent Offenders - Appraising and Managing Risk. American Psychological Association, Washington, DC.

[58] Hare, R. D. (2003). Manual for the Hare Psychopathy ChecklistRevised: 2nd Edition. MultiHealth Systems, Toronto, Canada.

[59] Chawla, N. V., Bowyer, K. W., Hall, L. O., \& Kegelmeyer, W. P. (2002). SMOTE: Synthetic Minority Over-sampling Technique. Journal of Artificial Intelligence Research, 16: 321-357.

[60] Tsamardinos, I., Aliferis, C. F., Statnikov, A., Brown, L. E. (2003). Scaling-up Bayesian Network Learning to Thousands of Variables Using Local Learning Technique. Technical Report DSL-TR-03-02, Mar. 2003.
[61] He, H., \& Garcia, E. A. (2009). Learning from Imbalanced Data. IEEE Transactions on Knowledge and Data Engineering, 21(9), 1263-1284.

[62] Constantinou, A. C., Yet, B., Fenton, N., Neil, M., \& Marsh, W. (2015). Value of Information analysis for Interventional and Counterfactual Bayesian networks in Forensic Medical Sciences. To appear in Artificial Intelligence in Medicine, 2015.

[63] de Campos, C. P., \& Ji, Q. (2011). Efficient Structure Learning of Bayesian Networks using Constraints. Journal of Machine Learning Research, 12: 663-689.

[64] Fenton, N. (2014). Effective Bayesian Modelling with Knowledge Before Data (Short Name: BAYES-KNOWLEDGE) https://www.eecs.qmul.ac.uk/ norman/projects/B_Knowledge.ht ml (Accessed: 7 October, 2015). 
APPENDIX A: The BN structure for DSVM-P \& DSVM-MSS studies

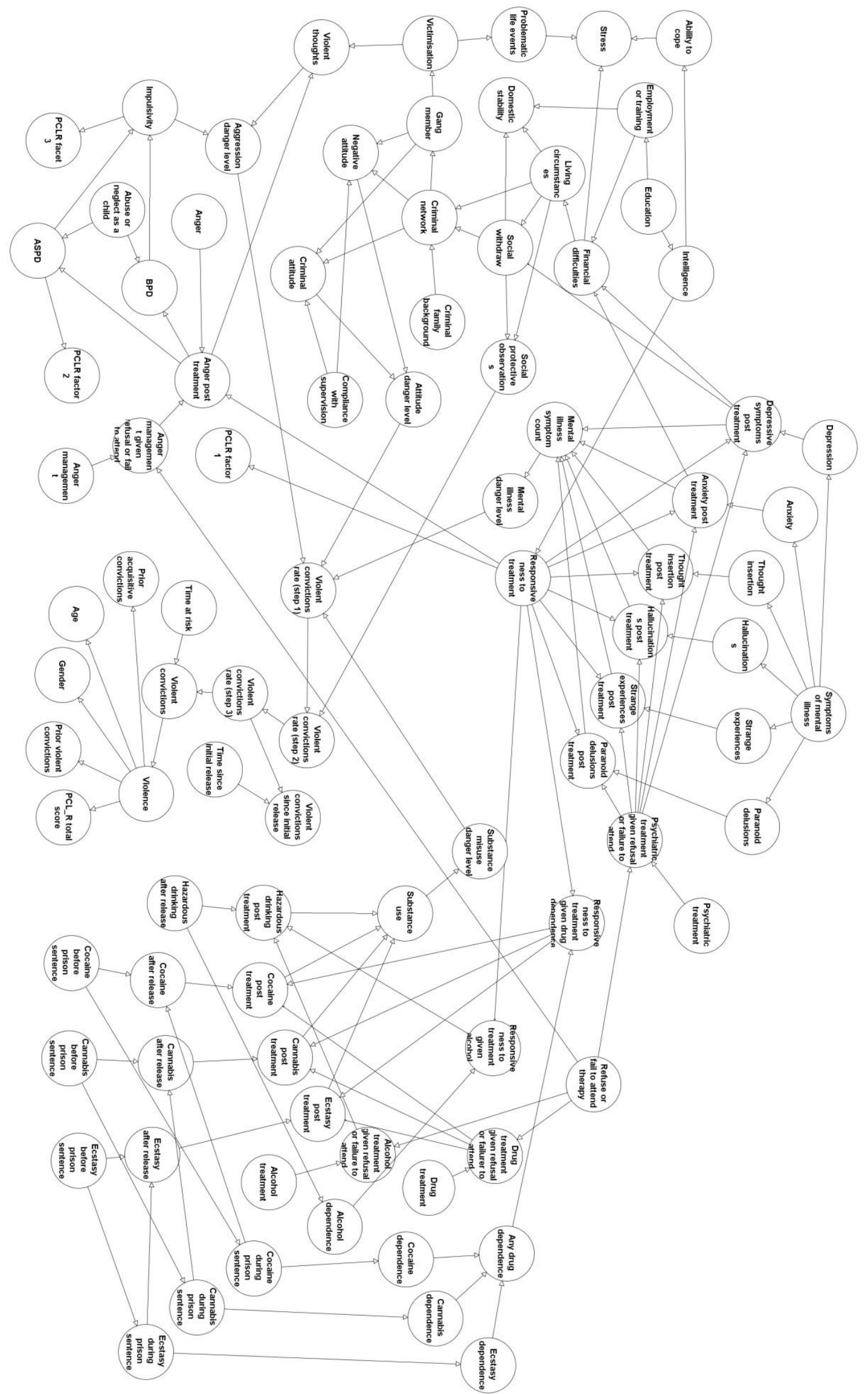

Figure A.1. The structure of the BN model from the DSVM-P study [10]. 


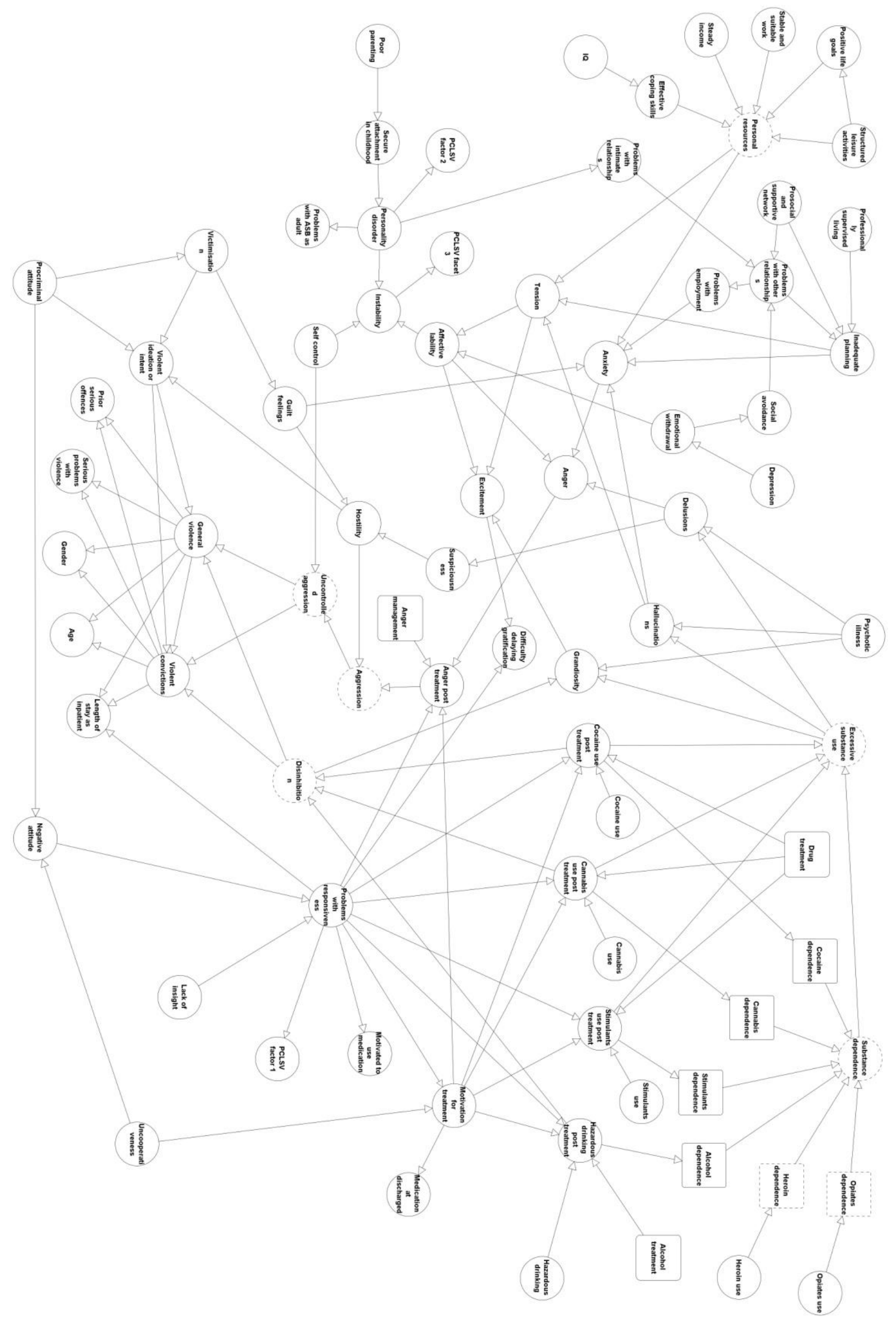

Figure A.2. The structure of the DSVM-MSS study [11]. 


\section{APPENDIX B: The BN model variables that have been downgraded in terms of complexity}

Table B.1. The BN model variables, from both the DSVM-P and DSVM-MSS studies, that have been downgraded, in terms of complexity, in order to reduce the risk of model overfitting as a result of limited data.

\begin{tabular}{|c|c|c|c|}
\hline Model & Model variable & Values from data & States in the BN \\
\hline DSVM-MSS & Age & 18 to 64 years & 18-21/22-25/26-29/30-34/35-39/40-49/50-59/60+ \\
\hline DSVM-MSS & Prior serious offences & 0 to $\max$ & None/One/2+ \\
\hline DSVM-MSS & Length of stay as inpatient & 7 to 7299 days & Up to 1 year/Up to 2 years/Up to 5 years/5+ years \\
\hline DSVM-MSS & PCLSVF1 & Score 1 to 12 & Low $=0-1$, Medium $=2-5$, High $=6+$ \\
\hline DSVM-MSS & PCLSVF2 & Score 1 to 12 & Low $=0-1$, Medium $=2-5$, High $=6+$ \\
\hline DSVM-MSS & PCLSVF3 & Score 1 to 6 & Low $=0-1$, Medium $=2-3$, High $=4+$ \\
\hline DSVM-MSS & IQ & Score 0 to $\max$ & Low average/Average/High average \\
\hline DSVM-MSS & Uncooperativeness & Score from 1 to 7 & $\mathrm{No}=1$, Partly $=2-4, \mathrm{Yes}=5+$ \\
\hline DSVM-MSS & Social avoidance & Score from 1 to 7 & $\mathrm{No}=1$, Partly $=2-4, \mathrm{Yes}=5+$ \\
\hline DSVM-MSS & Tension & Score from 1 to 7 & $\mathrm{No}=1$, Partly $=2-4, \mathrm{Yes}=5+$ \\
\hline DSVM-MSS & Guilt feelings & Score from 1 to 7 & $\mathrm{No}=1$, Partly $=2-4, \mathrm{Yes}=5+$ \\
\hline DSVM-MSS & Affective lability & Score from 1 to 7 & $\mathrm{No}=1$, Partly $=2-4, \mathrm{Yes}=5+$ \\
\hline DSVM-MSS & Anger & Score from 1 to 7 & $\mathrm{No}=1$, Partly $=2-4, \mathrm{Yes}=5+$ \\
\hline DSVM-MSS & Excitement & Score from 1 to 7 & $\mathrm{No}=1$, Partly $=2-4, \mathrm{Yes}=5+$ \\
\hline DSVM-MSS & Suspiciousness & Score from 1 to 7 & $\mathrm{No}=1$, Partly $=2-4, \mathrm{Yes}=5+$ \\
\hline DSVM-MSS & Hostility & Score from 1 to 7 & $\mathrm{No}=1$, Partly $=2-4, \mathrm{Yes}=5+$ \\
\hline DSVM-MSS & $\begin{array}{c}\text { Difficulty delaying } \\
\text { gratification }\end{array}$ & Score from 1 to 7 & $\mathrm{No}=1$, Partly $=2-4, \mathrm{Yes}=5+$ \\
\hline DSVM-MSS & Emotional withdrawal & Score from 1 to 7 & $\mathrm{No}=1$, Partly $=2-4, \mathrm{Yes}=5+$ \\
\hline DSVM-MSS & Delusions & Score from 1 to 7 & $\mathrm{No}=1$, Partly=2-4, Yes=5+ \\
\hline DSVM-MSS & Hallucination & Score from 1 to 7 & $\mathrm{No}=1$, Partly $=2-4, \mathrm{Yes}=5+$ \\
\hline DSVM-MSS & Grandiosity & Score from 1 to 7 & $\mathrm{No}=1$, Partly $=2-4, \mathrm{Yes}=5+$ \\
\hline DSVM-MSS & Anxiety & Score from 1 to 7 & $\mathrm{No}=1$, Partly $=2-4, \mathrm{Yes}=5+$ \\
\hline DSVM-MSS & Depression & Score from 1 to 7 & $\mathrm{No}=1$, Partly $=2-4, \mathrm{Yes}=5+$ \\
\hline DSVM-P & Age & 18 to 75 years & $18-19 / 20-21 / 22-25 / 26-29 / 30-34 / 35-39 / 40-49 / 50-59 / 60+$ \\
\hline DSVM-P & Domestic stability & $\begin{array}{l}\text { a) Frequent address change: } \\
\text { Integer, } \\
\text { b) Eviction: Boolean, } \\
\text { c) Family/friends } \\
\text { unsupportive: Score } 1 \text { to } 10 .\end{array}$ & $\begin{array}{l}\text { a) Frequent address change: } \mathrm{No}=0-3, \mathrm{Yes}=4+\text {, } \\
\text { b) Eviction: No=false, Yes=true, } \\
\text { c) Family/friends unsupportive: } \mathrm{No}=0-3, \mathrm{Yes}=4+\text {. } \\
\text { Rule introduced: if at least two of the three variables above } \\
\text { returns Yes, then Domestic stability }=\text { Low, otherwise Domestic } \\
\text { stability=High. }\end{array}$ \\
\hline DSVM-P & Financial difficulties & $\begin{array}{l}\text { a) Behind paying bills: Boolean, } \\
\text { b) Services cut off: Boolean, } \\
\text { c) Low income: Score from } 0 \text { to } \\
\text { max. }\end{array}$ & $\begin{array}{l}\text { a) Behind paying bills: } \mathrm{No}=\text { false, Yes=true, } \\
\text { b) Services cut off: } \mathrm{No}=\text { false, } \mathrm{Yes}=\text { true, } \\
\text { c) Low income: } \mathrm{No}=50+, \mathrm{Yes}=0-49 \text {. } \\
\text { Rule introduced: if at least one of the three variables above } \\
\text { returns Yes, then Financial difficulties }=Y e s \text {, otherwise Financial } \\
\text { difficulties }=\text { No. }\end{array}$ \\
\hline DSVM-P & Hazardous drinking & $\begin{array}{c}\text { Alcohol use disorder } \\
\text { identification test (AUDIT): Score } \\
0 \text { to } 32 .\end{array}$ & $\mathrm{No}=0-1, \mathrm{Yes}=8+$ \\
\hline DSVM-P & Problematic life events & $\begin{array}{l}\text { a) Separation/divorce: Boolean, } \\
\text { b) Problems with } \\
\text { friends/family/neighbour: } \\
\text { Boolean, } \\
\text { c) Redundant/sucked: Boolean. }\end{array}$ & $\begin{array}{l}\text { a) Separation/divorce: } \mathrm{No}=\text { false, Yes=true, } \\
\text { b) Problems with friends/family/neighbour: No=false, } \\
\text { Yes=true, } \\
\text { c) Redundant/sucked: } \mathrm{No}=\text { false, Yes=true, } \\
\text { Rule introduced: if at least one of the three variables above } \\
\text { returns Yes, then Problematic life events }=\text { Yes, otherwise } \\
\text { Problematic life events }=\text { No. }\end{array}$ \\
\hline DSVM-P & Stress & High stress score 0 to $\max$ & $\mathrm{No}=0-17, \mathrm{Yes}=18+$ \\
\hline DSVM-P & Intelligence & IQ Score 0 to 130 & $\begin{array}{c}\text { Extremely Low/ Borderline/ Low Average/Average/ High } \\
\text { Average/Superior }\end{array}$ \\
\hline DSVM-P & Criminal network & $\begin{array}{l}\text { a) Family/friends have criminal } \\
\text { convictions: Boolean, } \\
\text { b) Family/friends offered }\end{array}$ & $\begin{array}{l}\text { a) Family/friends have criminal convictions: No=false, } \\
\text { Yes=true, } \\
\text { b) Family/friends offered drugs: No=false, Yes=true, }\end{array}$ \\
\hline
\end{tabular}




\begin{tabular}{|c|c|c|c|}
\hline & & $\begin{array}{l}\text { drugs: Boolean, } \\
\text { c) Family/friends asked for } \\
\text { money/goods through crime: } \\
\text { Boolean, } \\
\text { d) Family/friends suggested to } \\
\text { commit a crime: Boolean, } \\
\text { e) Family/friends got into a } \\
\text { fight: Boolean. }\end{array}$ & $\begin{array}{l}\text { c) Family/friends asked for money/goods through crime: } \\
\text { No=false, Yes=true, } \\
\text { d) Family/friends suggested to commit a crime: No=false, } \\
\text { Yes=true, } \\
\text { e) Family/friends got into a fight: No=false, Yes=true, } \\
\text { Rule introduced: if at least one of the five variables above } \\
\text { returns Yes, then Criminal network=Yes, otherwise Criminal } \\
\text { network=No. }\end{array}$ \\
\hline DSVM-P & Criminal attitude & $\begin{array}{l}\text { a) OK to steal if very poor: } \\
\text { Score } 1 \text { to } 5 \text {, } \\
\text { b) OK to steal from the rick: } \\
\text { Score } 1 \text { to } 5 \text {, } \\
\text { c) OK to steal from shops that } \\
\text { make lots of money: Score } 1 \\
\text { to } 5, \\
\text { d) Sometimes it is OK to break } \\
\text { the law: Score } 1 \text { to } 5 \text {, }\end{array}$ & $\begin{array}{l}\text { a) OK to steal if very poor: } \mathrm{No}=0-1, \mathrm{Yes}=2+\text {, } \\
\text { b) OK to steal from the rick: } \mathrm{No}=0-1, \mathrm{Yes}=2+\text {, } \\
\text { c) OK to steal from shops that make lots of money: } \mathrm{No}=0-1 \text {, } \\
\text { Yes }=2+\text {, } \\
\text { d) Sometimes it is } \mathrm{OK} \text { to break the law: } \mathrm{No}=0-1, \mathrm{Yes}=2+\text {. } \\
\text { Rule introduced: if at least one of the four variables above } \\
\text { returns Yes, then Criminal attitude=Yes, otherwise Criminal } \\
\text { attitude=No. }\end{array}$ \\
\hline DSVM-P & Victimisation & $\begin{array}{l}\text { a) Victim of theft/burglary: } \\
\text { Boolean, } \\
\text { b) Victim of threats: Boolean, } \\
\text { c) Assaulted: Boolean. }\end{array}$ & $\begin{array}{l}\text { a) Victim of theft/burglary: } \mathrm{No}=\text { false, Yes }=\text { true, } \\
\text { b) Victim of threats: } \mathrm{No}=\text { false, Yes=true, } \\
\text { c) Assaulted: No=false, Yes=true. } \\
\text { Rule introduced: if at least one of the three variables above } \\
\text { returns Yes, then Criminal network }=Y e s \text {, otherwise Criminal } \\
\text { network }=\text { No. }\end{array}$ \\
\hline DSVM-P & Compliance with supervision & $\begin{array}{l}\text { Number of appointments missed } \\
\text { with probation officer: Score } 0 \text { to } \\
\text { max }\end{array}$ & $\mathrm{No}=1+, \mathrm{Yes}=0$ \\
\hline DSVM-P & Anger & STAXI trait score: Score 0 to 27 & $\mathrm{No}=0-2, \mathrm{Yes}=3+$. \\
\hline DSVM-P & $\begin{array}{l}\text { Cocaine (applies to all three } \\
\text { cocaine variables; before, } \\
\text { during and post-release) }\end{array}$ & $\begin{array}{l}\text { a) Cocaine powder: Boolean, } \\
\text { b) Crack cocaine: Boolean, }\end{array}$ & $\begin{array}{l}\text { a) Cocaine powder: } \mathrm{No}=\text { false, } Y \mathrm{es}=\text { true, } \\
\text { b) Crack cocaine: No=false, Yes=true, } \\
\text { Rule introduced: if at least one of the two variables above } \\
\text { returns Yes, then Cocaine }=Y e s \text {, otherwise Cocaine }=\text { No. }\end{array}$ \\
\hline DSVM-P & Responsiveness to treatment & $\begin{array}{l}\text { a) Not taken medication: } \\
\text { Boolean, } \\
\text { b) Missed injections: Boolean, }\end{array}$ & $\begin{array}{l}\text { a) Not taken medication: } \mathrm{No}=\text { false, Yes=true, } \\
\text { b) Missed injections: No=false, Yes=true, } \\
\text { Rule introduced: if one of the two variables above returns Yes, } \\
\text { then Responsiveness to treatment=Partly, if two of the variables } \\
\text { above return Yes, then Responsiveness to treatment=No, } \\
\text { otherwise Responsiveness to treatment }=\text { Yes. }\end{array}$ \\
\hline DSVM-P & PCLR Total score & PCL-R: Score 0 to 35 & $0-9 / 10-16 / 17-26 / 27+$ \\
\hline DSVM-P & Prior convictions & Score 0 to $\max$ & $0 / 1 / 2-5 / 6+$ \\
\hline DSVM-P & $\begin{array}{c}\text { Prior acquisitive crime } \\
\text { convictions }\end{array}$ & Score 0 to $\max$ & $0-2 / 3-12 / 13+$ \\
\hline
\end{tabular}

